\title{
On a Nonsymmetric Keyfitz-Kranzer System of Conservation Laws with Generalized and Modified Chaplygin Gas Pressure Law
}

\author{
Hongjun Cheng and Hanchun Yang \\ Department of Mathematics, Yunnan University, Kunming, Yunnan 650091, China \\ Correspondence should be addressed to Hongjun Cheng; hjcheng@ynu.edu.cn
}

Received 2 June 2013; Revised 20 October 2013; Accepted 25 October 2013

Academic Editor: Stephen C. Anco

Copyright (c) $2013 \mathrm{H}$. Cheng and H. Yang. This is an open access article distributed under the Creative Commons Attribution License, which permits unrestricted use, distribution, and reproduction in any medium, provided the original work is properly cited.

This paper is devoted to the study of a nonsymmetric Keyfitz-Kranzer system of conservation laws with the generalized and modified Chaplygin gas pressure law, which may admit delta shock waves, a topic of interest. Firstly, we solve the Riemann problems with piecewise constant data having a single discontinuity. For the generalized Chaplygin gas pressure law, the solution consists of three different structures: $R+J, S+J$, and $\delta$. Existence and uniqueness of delta shock solution are established under the generalized Rankine-Hugoniot relation and entropy condition. For the modified Chaplygin gas pressure law, the structures of solution are $R+J$ and $S+J$. Secondly, we discuss the limits of Riemann solutions for the modified Chaplygin gas pressure law as the pressure law tends to the generalized Chaplygin gas one. In particular, for some cases, the solution $S+J$ tends to a delta shock wave, and it is different from the delta shock wave for the generalized Chaplygin gas pressure law with the same initial data. Thirdly, we simulate the Riemann solutions and examine the formation process of delta shock wave by employing the Nessyahu-Tadmor scheme. The numerical results are coincident with the theoretical analysis.

\section{Introduction}

Nonlinear hyperbolic conservation laws are a fundamental principle in building mathematical models for many natural processes. For them, there exists an important kind of nonclassical solution, that is, delta shock wave. It is a generalization of an ordinary shock. From the mathematical point of view, it is a kind of discontinuity, on which at least one of the state variables contains Dirac delta function with a shock as its support. From the physical point of view, it represents the process of concentration of the mass. The theory of nonlinear hyperbolic conservation laws admitting delta shock waves is interesting and has been extensively developed in the last several years; see [1-13] and the references cited therein.

Consider the hyperbolic system of conservation laws:

$$
\begin{gathered}
\rho_{t}+(\rho(u-p))_{x}=0, \\
(\rho u)_{t}+(\rho u(u-p))_{x}=0,
\end{gathered}
$$

where $p=p(\rho)$ and $\rho \geq 0$. It belongs to the nonsymmetric Keyfitz-Kranzer system (see [14, 15]):

$$
\begin{gathered}
\rho_{t}+\left(\rho \phi\left(\rho, u_{1}, u_{2}, \ldots, u_{n}\right)\right)_{x}=0, \\
\left(\rho u_{i}\right)_{t}+\left(\rho u_{i} \phi\left(\rho, u_{1}, u_{2}, \ldots, u_{n}\right)\right)_{x}=0, \\
i=1,2, \ldots, n,
\end{gathered}
$$

which is of interest because it arises in such areas as elasticity theory, magnetohydrodynamics, and enhanced oil recovery. For delta shock waves, the nonsymmetric form is more convenient than the symmetric form (see [14]). Model (1) is also a transformation of the traffic flow model introduced by Aw and Rascle [16], where $\rho$ and $u>0$ are the density and velocity of cars on the roadway and $p$ is the velocity offset.

Let us recall the linear degeneracy and genuine nonlinearity of characteristic fields for quasilinear hyperbolic systems. Assume that $\lambda_{k}$ is the $k$ th eigenvalue of a quasilinear hyperbolic system and $r_{k}$ is the corresponding eigenvector. 
Then the $k$ th characteristic field is said to be genuinely nonlinear if $\nabla \lambda_{k} \cdot r_{k} \neq 0$, while if, on the other hand, $\nabla \lambda_{k} \cdot r_{k} \equiv$ 0 , we call it linearly degenerate (see $[17,18])$. For $(1)$, one can easily calculate that the eigenvalues are

$$
\lambda_{1}=u-p, \quad \lambda_{2}=u-p-\rho p^{\prime}
$$

and the corresponding right eigenvectors are

$$
r_{1}=\left(1, p^{\prime}\right), \quad r_{2}=(1,0) .
$$

Then

$$
\nabla \lambda_{1} \cdot r_{1} \equiv 0, \quad \nabla \lambda_{2} \cdot r_{2}=-\left(\rho p^{\prime \prime}+2 p^{\prime}\right) .
$$

Therefore the first characteristic field is always linearly degenerate and the second one is linearly degenerate or genuinely nonlinear depending on the behaviors of $p(\rho)$.

In general, a solution to system (1) strongly depends on the function $p(\rho)$. In [15], it is required that

$$
\begin{gathered}
p(0)=0, \\
\lim _{\rho \rightarrow 0} \rho p^{\prime}(\rho)=0, \\
\rho p^{\prime \prime}(\rho)+2 p^{\prime}(\rho)>0 \\
\text { for } \rho>0, \\
\lim _{\rho \rightarrow 0} \rho p(\rho)=0, \\
\lim _{\rho \rightarrow \infty}(\rho p(\rho))^{\prime} \geq A \geq 0, \\
\rho p^{\prime \prime}(\rho)+2 p^{\prime}(\rho)>0 \\
\text { for } \rho>0 .
\end{gathered}
$$

A prototype function satisfying (6) or (7) is $p=\rho^{\gamma}$, $\gamma>0$, with which the second characteristic $\lambda_{2}$ is genuinely nonlinear. Recently, in [19], Cheng introduced the function

$$
p=-\frac{B}{\rho}, \quad B>0,
$$

which is the prototype function satisfying

$$
\rho p^{\prime \prime}(\rho)+2 p^{\prime}(\rho) \equiv 0 \text { for } \rho>0 .
$$

At this moment, both eigenvalues are linearly degenerate; that is, this is a fully linearly degenerate system. The overlapping of linear degenerate characteristics results in the formation of delta shock waves.

In the present paper, we continue to study the system (1). We extend (8) to the function

$$
p=-\frac{B}{\rho^{\alpha}}, \quad 0<\alpha<1
$$

which does not satisfy (9). For the Riemann problem with initial data

$$
(u, \rho)(t=0, x)= \begin{cases}\left(u_{-}, \rho_{-}\right), & x<0 \\ \left(u_{+}, \rho_{+}\right), & x>0\end{cases}
$$

with the analysis method in phase plane, we construct solutions which are $R+J$ when $u_{+}+\left(B / \rho_{+}^{\alpha}\right) \geq u_{-}+\left(B / \rho_{-}^{\alpha}\right)$ and $S+J$ when $u_{-}<u_{+}+\left(B / \rho_{+}^{\alpha}\right)<u_{-}+\left(B / \rho_{-}^{\alpha}\right)$. However, for the rest case $u_{+}+\left(B / \rho_{+}^{\alpha}\right) \leq u_{-}$, the solution cannot be constructed by classical waves and delta shock waves should occur. Both existence and uniqueness of solutions involving delta shock waves are obtained by solving the generalized Rankine-Hugoniot relation under entropy condition.

We also introduce the function

$$
p=A \rho-\frac{B}{\rho^{\alpha}}, \quad A>0 .
$$

For the Riemann problem with initial data (11), by the analysis in phase plane, only two kinds of classical solutions $R+J$ and $S+J$ are constructed.

Noticing that (12) formally becomes (10) as $A \rightarrow 0$, we are interested in the topic that the limits of Riemann solutions to (1) and (12) are whether or not the Riemann solutions to (1) and (10). It is rigorously shown that, when $A \rightarrow 0$, some Riemann solutions containing $S$ and $J$ tend to a delta shock solution. However, its weight and speed are different from those of delta shock solution to (1), (10) with the same initial data (11). Besides, it is also proven that the rest Riemann solutions tend to just the ones of (1), (10) with the same initial data (11).

To confirm the theoretical analysis, we simulate the Riemann solutions and the formation process of delta shock waves by using the Nessyahu-Tadmor scheme [20]. The numerical results coincide completely with the theoretical analysis.

We remark that the functions (8), (10), and (12) are also the equations of state characterizing the standard, generalized, and modified Chaplygin gas, respectively, where $p$ is the pressure and $\rho$ the density. As everyone knows, the universe is experiencing an accelerated expansion. Usually, it is thought that the source of this acceleration is attributed to an exotic type of fluid called dark energy. Then people began to search for different candidates of the dark energy. Up to now, some theoretical models have been proposed. Among them, the standard, generalized, and modified Chaplygin gas are plausible (see [21-25]).

This paper is organized as follows. In Sections 2 and 3, we solve the Riemann problem for (1) with (10) and (12), respectively. In Section 4, we investigate the limits of solutions to (1), (12), and (11) as $A \rightarrow 0$. In Section 5, some numerical results are presented. Section 6 gives a conclusion.

\section{Riemann Problem for (1) with (10)}

In this section, we construct the solutions to system (1), (10) with initial data (11). The considered system has two eigenvalues:

$$
\lambda_{1}=u+\frac{B}{\rho^{\alpha}}, \quad \lambda_{2}=u+\frac{B(1-\alpha)}{\rho^{\alpha}}
$$

with corresponding right eigenvectors:

$$
r_{1}=\left(1, \frac{B \alpha}{\rho^{\alpha+1}}\right)^{T}, \quad r_{2}=(1,0)^{T}
$$


satisfying, respectively,

$$
\nabla \lambda_{1} \cdot r_{1} \equiv 0, \quad \nabla \lambda_{2} \cdot r_{2}=-\frac{B \alpha(1-\alpha)}{\rho^{\alpha+1}} .
$$

Therefore the system is strictly hyperbolic. The first wave family is linearly degenerate and the second one is genuinely nonlinear.

As usual, we seek the self-similar solution:

$$
(u, \rho)(t, x)=(u, \rho)(\xi), \quad \xi=\frac{x}{t},
$$

for which (1) with (10) becomes

$$
\begin{gathered}
-\xi \rho_{\xi}+\left(\rho\left(u+\frac{B}{\rho^{\alpha}}\right)\right)_{\xi}=0, \\
-\xi(\rho u)_{\xi}+\left(\rho u\left(u+\frac{B}{\rho^{\alpha}}\right)\right)_{\xi}=0
\end{gathered}
$$

and (11) changes into the infinity boundary condition

$$
(u, \rho)( \pm \infty)=\left(u_{ \pm}, \rho_{ \pm}\right) .
$$

For any smooth solutions, (17) is equivalent to

$$
\left(\begin{array}{cc}
u+\frac{B(1-\alpha)}{\rho^{\alpha}}-\xi & \rho \\
0 & u+\frac{B}{\rho^{\alpha}}-\xi
\end{array}\right)\left(\begin{array}{l}
d \rho \\
d u
\end{array}\right)=0,
$$

which provides either the general solution (constant state)

$$
(u, \rho)=\text { Const. } \quad(\rho>0)
$$

or singular solution, which is a wave of the first characteristic family,

$$
\begin{aligned}
& \xi=\lambda_{1}=u+\frac{B}{\rho^{\alpha}}, \\
& d\left(u+\frac{B}{\rho^{\alpha}}\right)=0,
\end{aligned}
$$

or rarefaction wave, which is a wave of the second characteristic family,

$$
\begin{gathered}
\xi=\lambda_{2}=u+\frac{B(1-\alpha)}{\rho^{\alpha}}, \\
d u=0 .
\end{gathered}
$$

Integrate (21) to obtain

$$
\begin{gathered}
\xi=u+\frac{B}{\rho^{\alpha}}, \\
u+\frac{B}{\rho^{\alpha}}=u_{-}+\frac{B}{\rho_{-}^{\alpha}},
\end{gathered}
$$

which is actually a contact discontinuity (see (26)). We also integrate (22) and take the requirement $\lambda_{2}\left(u_{-}, \rho_{-}\right)<\lambda_{2}(u, \rho)$ into account to obtain

$$
R:\left\{\begin{array}{l}
\xi=u+\frac{B(1-\alpha)}{\rho^{\alpha}}, \\
u=u_{-}, \quad \rho_{-}>\rho .
\end{array}\right.
$$

For a bounded discontinuity at $\xi=\sigma$, the RankineHugoniot relation

$$
\begin{gathered}
-\sigma[\rho]+\left[\rho\left(u+\frac{B}{\rho^{\alpha}}\right)\right]=0, \\
-\sigma[\rho u]+\left[\rho u\left(u+\frac{B}{\rho^{\alpha}}\right)\right]=0
\end{gathered}
$$

holds, where and in what follows $[a]=a_{-}-a$ denotes the jump of of $a$ across the discontinuity. By solving (25), we obtain contact discontinuity, which is a wave of the first characteristic family,

$$
J:\left\{\begin{array}{l}
\sigma=u+\frac{B}{\rho^{\alpha}}, \\
u+\frac{B}{\rho^{\alpha}}=u_{-}+\frac{B}{\rho_{-}^{\alpha}},
\end{array}\right.
$$

or shock wave, which is a wave of the second characteristic family,

$$
S:\left\{\begin{array}{l}
\sigma=u+\frac{\rho_{-} \cdot\left(B / \rho_{-}^{\alpha}\right)-\rho \cdot\left(B / \rho^{\alpha}\right)}{\rho_{-}-\rho} \\
u=u_{-} .
\end{array}\right.
$$

The stability condition (entropy condition) for shocks can be defined as "three incoming, one outgoing," which means that three of the characteristic lines on both sides of shock, one $\lambda_{1}$ and two $\lambda_{2}$, are incoming with respect to the shock, while the remaining one, $\lambda_{1}$, is outgoing. The stability condition is equivalent to

$$
\rho_{-}<\rho .
$$

The curves in $(u, \rho)$-plane expressed by the second equation in (24), (26), and (27) are called as the rarefaction wave curve, contact discontinuity curve, and shock wave curve, respectively. For the contact discontinuity curve, we have $u_{\rho}=(B \alpha) / \rho^{\alpha+1}>0$ and $u_{\rho \rho}=-B \alpha(\alpha+1) / \rho^{\alpha+2}<$ 0 , which mean that the curve is monotonic increasing and concave; moreover, it can be proved that $\lim _{\rho \rightarrow 0} u=-\infty$ and $\lim _{\rho \rightarrow+\infty} u=u_{-}+\left(B / \rho_{-}^{\alpha}\right)$, which show that the curve has $\rho=0$ and $u=u_{-}+\left(B / \rho_{-}^{\alpha}\right)$ as its two asymptotes.

Fixing a left state $\left(u_{-}, \rho_{-}\right)$, we draw the rarefaction wave curve, contact discontinuity curve, and shock wave curve. Also, we draw the curve $u+\left(B / \rho^{\alpha}\right)=u_{-}$. Then the phase plane is divided into three domains $I\left(u_{-}, \rho_{-}\right), I I\left(u_{-}, \rho_{-}\right)$and $I I I\left(u_{-}, \rho_{-}\right)$by the curves $u+\left(B / \rho^{\alpha}\right)=u_{-}+\left(B / \rho_{-}^{\alpha}\right)$, and $u+\left(B / \rho^{\alpha}\right)=u_{-}$(see Figure 1):

$$
\begin{gathered}
I\left(u_{-}, \rho_{-}\right)=\left\{(u, \rho) \mid \rho>0, u+\frac{B}{\rho^{\alpha}} \geq u_{-}+\frac{B}{\rho_{-}^{\alpha}}\right\}, \\
I I\left(u_{-}, \rho_{-}\right)=\left\{(u, \rho) \mid \rho>0, u_{-}<u+\frac{B}{\rho^{\alpha}}<u_{-}+\frac{B}{\rho_{-}^{\alpha}}\right\}, \\
I I I\left(u_{-}, \rho_{-}\right)=\left\{(u, \rho) \mid \rho>0, u+\frac{B}{\rho^{\alpha}} \leq u_{-}\right\} .
\end{gathered}
$$

Then by the analysis method in phase plane, using the classical elementary waves $R, S$, and $J$, one can construct the solutions of Riemann problem in the following cases: 


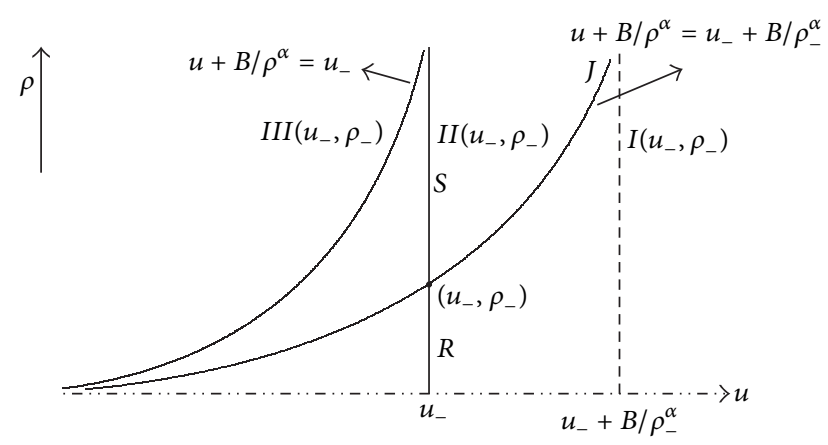

Figure 1: Phase plane and elementary wave curves.

(1) when $\left(u_{+}, \rho_{+}\right) \in I\left(u_{-}, \rho_{-}\right)$, the solution is $R+J$;

(2) when $\left(u_{+}, \rho_{+}\right) \in I I\left(u_{-}, \rho_{-}\right)$, the solution is $S+J$.

In these solutions, the intermediate state $\left(u_{*}, \rho_{*}\right)$ satisfies

$$
u_{*}=u_{-}, \quad u_{*}+\frac{B}{\rho_{*}^{\alpha}}=u_{+}+\frac{B}{\rho_{+}^{\alpha}} .
$$

However, for the case $\left(u_{+}, \rho_{+}\right) \in I I I\left(u_{-}, \rho_{-}\right)$, one can check that the Riemann solution cannot be constructed by the classical elementary waves. In fact, at this moment, the characteristic lines from initial data will overlap in a domain. So singularity must happen. It is easy to know that the singularity is impossible to be a jump with finite amplitude because the Rankine-Hugoniot relation is not satisfied on the bounded jump. In other words, there is no solution which is piecewise smooth and bounded.

We define a two-dimensional weighted delta function $w(s) \delta_{L}$ supported on a smooth curve $L$ parameterized as $t=$ $t(s)$, and $x=x(s)(c \leq s \leq d)$ by

$$
\left\langle w(s) \delta_{L}, \phi(x, t)\right\rangle=\int_{c}^{d} w(s) \phi(x(s), t(s)) d s
$$

for all $\phi \in C_{0}^{\infty}\left(R^{2}\right)$.

With this definition, motivated by $[4-6,10,12,19]$, it is well known that the Riemann solution should be the delta shock solution of the form

$$
(u, \rho)(t, x)= \begin{cases}\left(u_{-}, \rho_{-}\right), & x<\sigma t \\ (\sigma, w(t) \delta(x-\sigma t)), & x=\sigma t \\ \left(u_{+}, \rho_{+}\right), & x>\sigma t\end{cases}
$$

with $1 / \rho$ defined by (see $[10,12,26])$

$$
\frac{1}{\rho(t, x)}= \begin{cases}\frac{1}{\rho_{-}}, & x<\sigma t \\ 0, & x=\sigma t \\ \frac{1}{\rho_{+}}, & x>\sigma t\end{cases}
$$

which satisfies the generalized Rankine-Hugoniot relation

$$
\begin{aligned}
\frac{d w(t)}{d t} & =-\sigma[\rho]+\left[\rho\left(u+\frac{B}{\rho^{\alpha}}\right)\right], \\
\frac{d w(t) \sigma}{d t} & =-\sigma[\rho u]+\left[\rho u\left(u+\frac{B}{\rho^{\alpha}}\right)\right]
\end{aligned}
$$

and the entropy condition

$$
\lambda_{1}\left(\rho_{+}, u_{+}\right) \leq \sigma \leq \lambda_{2}\left(\rho_{-}, u_{-}\right),
$$

where $w(t)$ and $\sigma$ are the weight and velocity of delta shock wave, respectively.

In what follows, we establish the existence and uniqueness of solution to the generalized Rankine-Hugoniot relation (34) under the entropy condition (35).

From (34) with initial data $w(0)=0$, we obtain the algebra equations:

$$
\begin{gathered}
w(t)=-\sigma[\rho] t+\left[\rho\left(u+\frac{B}{\rho^{\alpha}}\right)\right] t, \\
w(t) \sigma=-\sigma[\rho u] t+\left[\rho u\left(u+\frac{B}{\rho^{\alpha}}\right)\right] t,
\end{gathered}
$$

from which it follows that

$$
[\rho] \sigma^{2}-\left(\left[\rho\left(u+\frac{B}{\rho^{\alpha}}\right)\right]+[\rho u]\right) \sigma+\left[\rho u\left(u+\frac{B}{\rho^{\alpha}}\right)\right]=0 .
$$

Entropy condition (35) also reads

$$
u_{+}+\frac{B}{\rho_{+}^{\alpha}} \leq \sigma \leq u_{-}+\frac{B(1-\alpha)}{\rho_{-}^{\alpha}}
$$

Set

$$
\begin{aligned}
F(\sigma)= & \text { the left-hand side of }(37) \\
= & \rho_{-}\left(\sigma-u_{-}\right)\left(\sigma-\left(u_{-}+\frac{B}{\rho_{-}^{\alpha}}\right)\right) \\
& -\rho_{+}\left(\sigma-u_{+}\right)\left(\sigma-\left(u_{+}+\frac{B}{\rho_{+}^{\alpha}}\right)\right) .
\end{aligned}
$$

We have

$$
\begin{aligned}
F^{\prime}(\sigma)= & \rho_{-}\left(\sigma-\left(u_{-}+\frac{B}{\rho_{-}^{\alpha}}\right)\right)+\rho_{-}\left(\sigma-u_{-}\right) \\
& -\rho_{+}\left(\sigma-\left(u_{+}+\frac{B}{\rho_{+}^{\alpha}}\right)\right)-\rho_{+}\left(\sigma-u_{+}\right) .
\end{aligned}
$$

Denote

$$
E_{1}=\left[u_{+}+\frac{B}{\rho_{+}^{\alpha}}, u_{-}\right], \quad E_{2}=\left(u_{-}, u_{-}+\frac{B(1-\alpha)}{\rho_{-}^{\alpha}}\right] .
$$

If $\sigma \in E_{2}$, we have $F(\sigma)<0$, which means that (37) has no solution in $E_{2}$. If $\sigma \in E_{1}$, we have

$$
F\left(u_{+}+\frac{B}{\rho_{+}^{\alpha}}\right)>0, \quad F\left(u_{-}\right)<0, \quad F^{\prime}(\sigma)<0,
$$

which can show that (37) has a unique solution in $E_{1}$ by zero point theorem in mathematical analysis. In summary, we can conclude that (37) has a unique solution under (38). Returning to (36), we can obtain $w(t)$ uniquely. 
Theorem 1. For the Riemann problem (1), (10), and (11), there exists a unique entropy solution, which consists of a rarefaction wave and a contact discontinuity when $u_{+}+\left(B / \rho_{+}^{\alpha}\right) \geq u_{-}+$ $\left(B / \rho_{-}^{\alpha}\right)$, a shock and a contact discontinuity when $u_{-}<u_{+}+$ $\left(B / \rho_{+}^{\alpha}\right)<u_{-}+\left(B / \rho_{-}^{\alpha}\right)$, and a delta shock wave when $u_{+}+$ $\left(B / \rho_{+}^{\alpha}\right) \leq u_{-}$.

\section{Riemann Problem for (1) with (12)}

In this section, we briefly solve Riemann problem (1), (12) and (11). The eigenvalues are

$$
\lambda_{1}=u-A \rho+\frac{B}{\rho^{\alpha}}, \quad \lambda_{2}=u-2 A \rho+\frac{B(1-\alpha)}{\rho^{\alpha}} .
$$

The corresponding right eigenvectors are

$$
r_{1}=\left(1, A+\frac{B \alpha}{\rho^{\alpha+1}}\right), \quad r_{2}=(1,0)^{T}
$$

Moreover,

$$
\nabla \lambda_{1} \cdot r_{1} \equiv 0, \quad \nabla \lambda_{2} \cdot r_{2}=-2 A-\frac{B \alpha(1-\alpha)}{\rho^{\alpha+1}}
$$

Thus the system is strictly hyperbolic. The first wave family is linearly degenerate and the second one is genuinely nonlinear.

One can check that, besides the constant state

$$
(u, \rho)=\text { Const., }
$$

the classical elementary waves consist of rarefaction waves

$$
R:\left\{\begin{array}{l}
\xi=\lambda_{1}=u-2 A \rho+\frac{B(1-\alpha)}{\rho^{\alpha}} \\
u=u_{-}, \quad \rho_{-}>\rho
\end{array}\right.
$$

contact discontinuities

$$
J:\left\{\begin{array}{l}
\tau=u-A \rho+\frac{B}{\rho^{\alpha}}, \\
u-A \rho+\frac{B}{\rho^{\alpha}}=u_{-}-A \rho_{-}+\frac{B}{\rho_{-}^{\alpha}},
\end{array}\right.
$$

and shocks

$$
S:\left\{\begin{array}{l}
\sigma=u-\frac{\rho_{-}\left(A \rho_{-}-\left(B / \rho_{-}^{\alpha}\right)\right)-\rho\left(A \rho-\left(B / \rho^{\alpha}\right)\right)}{\rho_{-}-\rho}, \\
u=u_{-}, \quad \rho_{-}<\rho .
\end{array}\right.
$$

In $(u, \rho)$-plane, from (48), for the contact discontinuity curve $u=A \rho-\left(B / \rho^{\alpha}\right)+u_{-}-A \rho_{-}+\left(B / \rho_{-}^{\alpha}\right)$, we have $u_{\rho}=A+\left(B \alpha / \rho^{\alpha+1}\right)>0$, and $u_{\rho \rho}=-B \alpha(\alpha+1) / \rho^{\alpha+2}<0$, which show that the contact discontinuity curve is monotonic increasing and concave. Moreover, we have $\lim _{\rho \rightarrow+\infty} u=+\infty$ and $\lim _{\rho \rightarrow 0} u=-\infty$.

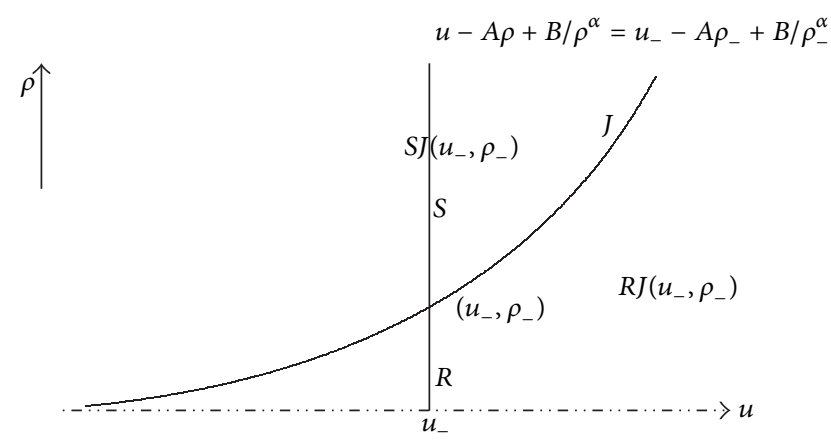

FIgURE 2: Phase plane and elementary wave curves.

For a given state $\left(u_{-}, \rho_{-}\right)$, the phase plane is divided into two domains $R J\left(u_{-}, \rho_{-}\right)$and $S J\left(u_{-}, \rho_{-}\right)$by the curves $u-A \rho+$ $\left(B / \rho^{\alpha}\right)=u_{-}-A \rho_{-}+\left(B / \rho_{-}^{\alpha}\right)$ (see Figure 2$)$ :

$$
\begin{aligned}
& R J\left(u_{-}, \rho_{-}\right) \\
& \quad=\left\{(u, \rho) \mid \rho>0, u-A \rho+\frac{B}{\rho^{\alpha}} \geq u_{-}-A \rho_{-}+\frac{B}{\rho_{-}^{\alpha}}\right\}, \\
& S J\left(u_{-}, \rho_{-}\right) \\
& \quad=\left\{(u, \rho) \mid \rho>0, u-A \rho+\frac{B}{\rho^{\alpha}}<u_{-}-A \rho_{-}+\frac{B}{\rho_{-}^{\alpha}}\right\} .
\end{aligned}
$$

Using the classical elementary waves, one can obtain the solutions of Riemann problem as follows:

(a) when $\left(u_{+}, \rho_{+}\right) \in R J\left(u_{-}, \rho_{-}\right)$, the solution is $R+J$;

(b) when $\left(u_{+}, \rho_{+}\right) \in S J\left(u_{-}, \rho_{-}\right)$, the solution is $S+J$.

In these solutions, the intermediate state $\left(u_{*}, \rho_{*}\right)$ satisfies

$$
u_{*}=u_{-}, \quad u_{*}-A \rho_{*}+\frac{B}{\rho_{*}^{\alpha}}=u_{+}-A \rho_{+}+\frac{B}{\rho_{+}^{\alpha}} .
$$

Theorem 2. For the Riemann problem (1), (12), and (11), there exists a unique entropy solution, which consists of a rarefaction wave and a contact discontinuity when $u-A \rho+\left(B / \rho^{\alpha}\right) \geq$ $u_{-}-A \rho_{-}+\left(B / \rho_{-}^{\alpha}\right)$ and $a$ shock and a contact discontinuity when $u-A \rho+\left(B / \rho^{\alpha}\right)<u_{-}-A \rho_{-}+\left(B / \rho_{-}^{\alpha}\right)$.

\section{Limits of Riemann Solutions to (1) with (12)}

In this section, we consider the limits of the Riemann solutions to (1), (12), and (11) as $A \rightarrow 0$ case by case.

Case 1. Consider $\left(u_{+}, \rho_{+}\right) \in I\left(u_{-}, \rho_{-}\right)$.

Lemma 3. When $\left(u_{+}, \rho_{+}\right) \in I\left(u_{-}, \rho_{-}\right)$, there exists $A_{0}>0$ such that $\left(u_{+}, \rho_{+}\right) \in R J\left(u_{-}, \rho_{-}\right)$when $0<A<A_{0}$.

Proof. All states $(u, \rho)$ connected with $\left(u_{-}, \rho_{-}\right)$by a contact discontinuity (48) satisfy

$$
u-A \rho+\frac{B}{\rho^{\alpha}}=u_{-}-A \rho_{-}+\frac{B}{\rho_{-}^{\alpha}} .
$$


If $\rho_{+} \leq \rho_{-}, A_{0}$ can be taken as arbitrary nonnegative real number. If $\rho_{+}>\rho_{-}$, by taking $A_{0}$ determined by

$$
u_{+}-A_{0} \rho_{+}+\frac{B}{\rho_{+}^{\alpha}}=u_{-}-A_{0} \rho_{-}+\frac{B}{\rho_{-}^{\alpha}},
$$

we have the conclusion.

For any $A_{0}>A>0$, the solution to Riemann problem (1), (11), and (12) is a rarefaction wave $R$ followed by a contact discontinuity $J$ with the intermediate state $\left(u_{*}^{A}, \rho_{*}^{A}\right)$ besides two constant states $\left(u_{-}, \rho_{-}\right)$and $\left(u_{+}, \rho_{+}\right)$. They have the following relations:

$$
\begin{gathered}
R:\left\{\begin{array}{l}
\xi=u-2 A \rho+\frac{B(1-\alpha)}{\rho^{\alpha}}, \\
u=u_{-}, \quad \rho_{-}>\rho>\rho_{*}^{A},
\end{array}\right. \\
J:\left\{\begin{array}{l}
\tau^{A}=u_{+}-A \rho_{+}+\frac{B}{\rho_{+}^{\alpha}}, \\
m u_{*}^{A}-A \rho_{*}^{A}+\frac{B}{\left(\rho_{*}^{A}\right)^{\alpha}}=u_{+}-A \rho_{+}+\frac{B}{\rho_{+}^{\alpha}} .
\end{array}\right.
\end{gathered}
$$

Denote $\left(u_{*}, \rho_{*}\right)=\lim _{A \rightarrow 0}\left(u_{*}^{A}, \rho_{*}^{A}\right)$. Letting $A \rightarrow 0$ in (55), then $R$ turns into

$$
R:\left\{\begin{array}{l}
\xi=u+\frac{B(1-\alpha)}{\rho^{\alpha}} \\
u=u_{-}, \quad \rho_{-}>\rho>\rho^{*}
\end{array}\right.
$$

and $J$ becomes

$$
J:\left\{\begin{array}{l}
\tau=u_{+}+\frac{B}{\rho_{+}^{\alpha}} \\
u_{*}+\frac{B}{\rho_{*}^{\alpha}}=u_{+}+\frac{B}{\rho_{+}^{\alpha}} .
\end{array}\right.
$$

One can see that the limit in this situation is the same as the solution to (1), (10) with the same initial data (11).

Case 2. Consider $\left(u_{+}, \rho_{+}\right) \in I I\left(u_{-}, \rho_{-}\right)$.

Lemma 4. When $\left(u_{+}, \rho_{+}\right) \in I I\left(u_{-}, \rho_{-}\right)$, there exists $A_{0}>0$ such that $\left(u_{+}, \rho_{+}\right) \in S J\left(u_{-}, \rho_{-}\right)$when $0<A<A_{0}$.

This lemma can be proved in a way completely similar to that of Lemma 3.

For any $A_{0}>A>0$, the solution of Riemann problem (1), (11), and (12) is a shock $S$ followed by a contact discontinuity $J$ with the intermediate state $\left(u_{*}^{A}, \rho_{*}^{A}\right)$ besides two constant states $\left(u_{-}, \rho_{-}\right)$and $\left(u_{+}, \rho_{+}\right)$. The solution can be expressed as

$$
\begin{gathered}
S:\left\{\begin{aligned}
\sigma^{A}= & u_{*}^{A} \\
& -\frac{\rho_{-}\left(A \rho_{-}-\left(B / \rho_{-}^{\alpha}\right)\right)-\rho_{*}^{A}\left(A \rho_{*}^{A}-\left(B /\left(\rho_{*}^{A}\right)^{\alpha}\right)\right)}{\rho_{-}-\rho_{*}^{A}}, \\
u_{*}^{A}= & u_{-}, \quad \rho_{-}<\rho_{*}^{A},
\end{aligned}\right. \\
J:\left\{\begin{array}{l}
\tau^{A}=u_{+}-A \rho_{+}+\frac{B}{\rho_{+}^{\alpha}}, \\
u_{*}^{A}-A \rho_{*}^{A}+\frac{B}{\left(\rho_{*}^{A}\right)^{\alpha}}=u_{+}-A \rho_{+}+\frac{B}{\rho_{+}^{\alpha}},
\end{array}\right.
\end{gathered}
$$

where $\sigma^{A}$ and $\tau^{A}$ are the propagation speeds of $S$ and $J$, respectively.
Denote $\left(u_{*}, \rho_{*}\right)=\lim _{A \rightarrow 0}\left(u_{*}^{A}, \rho_{*}^{A}\right)$. We have $\rho_{*} \neq+\infty$. In fact, if $\rho_{*}=+\infty$, from (58), we have

$$
u_{-}-\left(u_{+}+\frac{B}{\rho_{+}^{\alpha}}\right)=\lim _{A \rightarrow 0} A \rho_{*}^{A} \geq 0,
$$

which contradicts with $\left(u_{+}, \rho_{+}\right) \in I I\left(u_{-}, \rho_{-}\right)$, which is $u_{-}<$ $u_{+}+\left(B / \rho_{+}^{\alpha}\right)<u_{-}+\left(B / \rho_{-}^{\alpha}\right)$.

Letting $A \rightarrow 0$ in (58), then $S$ turns into

$$
S:\left\{\begin{array}{l}
\sigma=u_{*} \\
\quad-\frac{-\rho_{-} \cdot\left(B / \rho_{-}^{\alpha}\right)+\rho_{*} \cdot\left(B / \rho_{*}^{\alpha}\right)}{\rho_{-}-\rho_{*}} \\
u=u_{-}
\end{array}\right.
$$

and $J$ tends to

$$
J:\left\{\begin{array}{l}
\tau=u_{+}+\frac{B}{\rho_{+}^{\alpha}}, \\
u_{*}+\frac{B}{\rho_{*}^{\alpha}}=u_{+}+\frac{B}{\rho_{+}^{\alpha}} .
\end{array}\right.
$$

One can observe that the limit in this case is also the same as that in the solution to (1), (10) with the same initial data (11).

Case 3. Consider $\left(u_{+}, \rho_{+}\right) \in I I I\left(u_{-}, \rho_{-}\right)$.

For this case, analogous to Lemma 3, we have the following.

Lemma 5. When $\left(u_{+}, \rho_{+}\right) \in I I I\left(u_{-}, \rho_{-}\right)$, there exists $A_{0}>0$ such that $\left(u_{+}, \rho_{+}\right) \in S J\left(u_{-}, \rho_{-}\right)$when $0<A<A_{0}$.

When $0<A<A_{0}$, the solution of Riemann problem (1), (11), and (12) is a shock $S$ followed by a contact discontinuity $J$ with the intermediate state $\left(u_{*}^{A}, \rho_{*}^{A}\right)$ besides two constant states $\left(u_{-}, \rho_{-}\right)$and $\left(u_{+}, \rho_{+}\right)$. They have the following relations:

$$
\begin{gathered}
S:\left\{\begin{aligned}
\sigma^{A}= & u_{*}^{A} \\
& -\frac{\rho_{-}\left(A \rho_{-}-\left(B / \rho_{-}^{\alpha}\right)\right)-\rho_{*}^{A}\left(A \rho_{*}^{A}-\left(B /\left(\rho_{*}^{A}\right)^{\alpha}\right)\right)}{\rho_{-}-\rho_{*}^{A}} \\
u_{*}^{A}= & u_{-}, \quad \rho_{-}<\rho_{*}^{A},
\end{aligned}\right. \\
J:\left\{\begin{array}{l}
\tau^{A}=u_{+}-A \rho_{+}+\frac{B}{\rho_{+}^{\alpha}}, \\
u_{*}^{A}-A \rho_{*}^{A}+\frac{B}{\left(\rho_{*}^{A}\right)^{\alpha}}=u_{+}-A \rho_{+}+\frac{B}{\rho_{+}^{\alpha}}
\end{array}\right.
\end{gathered}
$$

where $\sigma^{A}$ and $\tau^{A}$ are the propagation speeds of $S$ and $J$, respectively. Then we have the following lemmas.

Lemma 6. One has $\lim _{A \rightarrow 0} \rho_{*}^{A}=+\infty$.

Proof. From the second equations in (62) and (63), we easily get

$$
u_{-}-A \rho_{*}^{A}+\frac{B}{\left(\rho_{*}^{A}\right)^{\alpha}}=u_{+}-A \rho_{+}+\frac{B}{\rho_{+}^{\alpha}}, \quad \rho_{*}^{A}>\rho_{-} .
$$




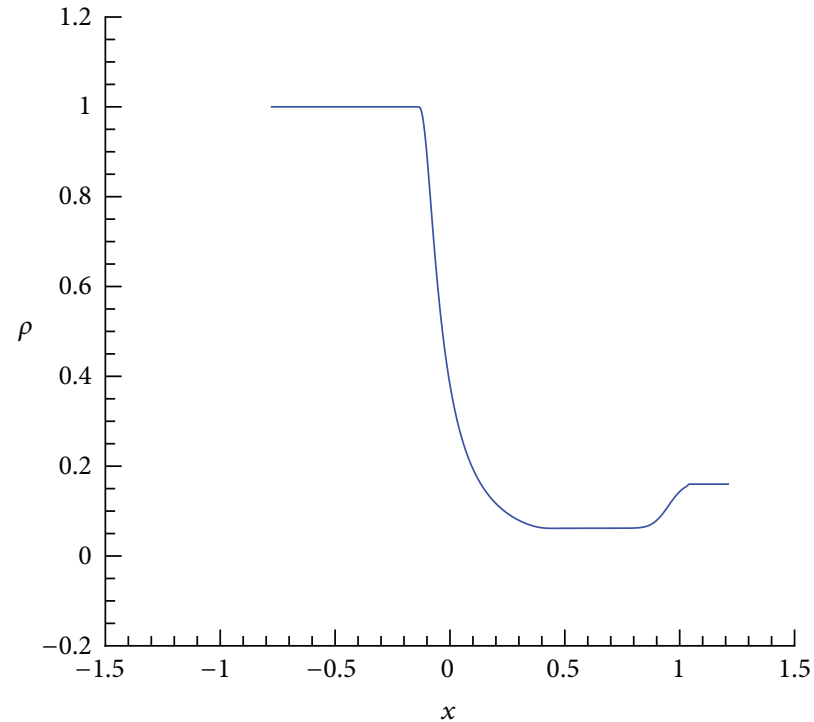

(a)

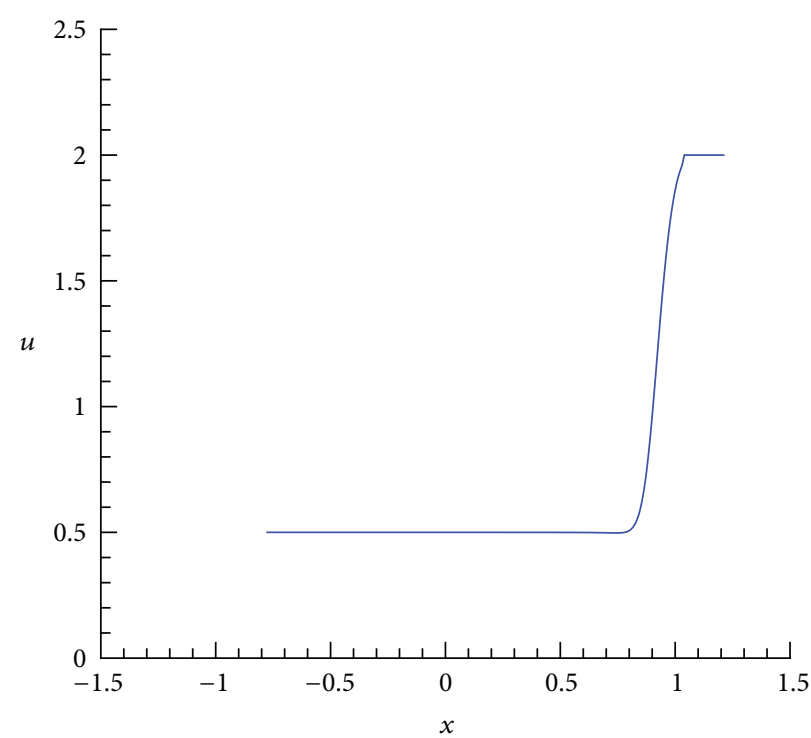

(b)

Figure 3: Numerical results for $R+J$.

If $\lim _{A \rightarrow 0} \rho_{*}^{A}=k \neq+\infty$, we have

$$
u_{-}+\frac{B}{k^{\alpha}}=u_{+}+\frac{B}{\rho_{+}^{\alpha}}
$$

which is impossible since $\left(u_{+}, \rho_{+}\right) \in \operatorname{III}\left(u_{-}, \rho_{-}\right)$; that is, $u_{+}+$ $\left(B / \rho_{+}^{\alpha}\right) \leq u_{-}$. Therefore, $\lim _{A \rightarrow 0} \rho_{*}^{A}=+\infty$.

Lemma 7. One has $\lim _{A \rightarrow 0} \sigma^{A}=\lim _{A \rightarrow 0} \tau^{A}=u_{+}+\left(B / \rho_{+}^{\alpha}\right):=$ $\sigma_{0}$.

Proof. From (62) and (63), we conclude that

$$
\begin{aligned}
& \lim _{A \rightarrow 0} \sigma^{A} \\
& =\lim _{A \rightarrow 0}\left(u_{*}^{A}-\frac{\rho_{-}\left(A \rho_{-}-\left(B / \rho_{-}^{\alpha}\right)\right)-\rho_{*}^{A}\left(A \rho_{*}^{A}-\left(B /\left(\rho_{*}^{A}\right)^{\alpha}\right)\right)}{\rho_{-}-\rho_{*}^{A}}\right) \\
& =\lim _{A \rightarrow 0}\left(u_{*}^{A}-\frac{A \rho_{-}-\left(B / \rho_{-}^{\alpha}\right)}{1-\left(\rho_{*}^{A} / \rho_{-}\right)}+\frac{A \rho_{*}^{A}-\left(B /\left(\rho_{*}^{A}\right)^{\alpha}\right)}{\left(\rho_{-} / \rho_{*}^{A}\right)-1}\right) \\
& =u_{+}+\frac{B}{\rho_{+}^{\alpha}}, \\
& \lim _{A \rightarrow 0} \tau^{A}=\lim _{A \rightarrow 0}\left(u_{+}-A \rho_{+}+\frac{B}{\rho_{+}^{\alpha}}\right)=u_{+}+\frac{B}{\rho_{+}^{\alpha}} .
\end{aligned}
$$

The proof is complete.

Lemmas 6-7 shows that when $A$ drops to zero, $S$ and $J$ coincide, and the intermediate density $\rho_{*}^{A}$ becomes singular.
Lemma 8. One has

$$
\begin{gathered}
\lim _{A \rightarrow 0}\left(\tau^{A}-\sigma^{A}\right) \rho_{*}^{A}=-\sigma_{0}[\rho]+\left[\rho\left(u+\frac{B}{\rho^{\alpha}}\right)\right], \\
\lim _{A \rightarrow 0}\left(\tau^{A}-\sigma^{A}\right) \rho_{*}^{A} u_{*}^{A}=-\sigma_{0}[\rho u]+\left[\rho u\left(u+\frac{B}{\rho^{\alpha}}\right)\right] .
\end{gathered}
$$

Proof. The Rankine-Hugoniot relations for $S$ and $J$ read, respectively,

$$
\begin{aligned}
& -\sigma^{A}\left(\rho_{-}-\rho_{*}^{A}\right)+\left(\rho_{-}\left(u_{-}-A \rho_{-}+\frac{B}{\rho_{-}^{\alpha}}\right)\right. \\
& \left.-\rho_{*}^{A}\left(u_{*}^{A}-A \rho_{*}^{A}+\frac{B}{\left(\rho_{*}^{A}\right)^{\alpha}}\right)\right)=0, \\
& -\sigma^{A}\left(\rho_{-} u_{-}-\rho_{*}^{A} u_{*}^{A}\right)+\left(\rho_{-} u_{-}\left(u_{-}-A \rho_{-}+\frac{B}{\rho_{-}^{\alpha}}\right)\right. \\
& \left.-\rho_{*}^{A} u_{*}^{A}\left(u_{*}^{A}-A \rho_{*}^{A}+\frac{B}{\left(\rho_{*}^{A}\right)^{\alpha}}\right)\right)=0, \\
& -\tau^{A}\left(\rho_{*}^{A}-\rho_{+}\right)+\left(\rho_{*}^{A}\left(u_{*}^{A}-A \rho_{*}^{A}+\frac{B}{\left(\rho_{*}^{A}\right)^{\alpha}}\right)\right. \\
& \left.-\rho_{+}\left(u_{+}-A \rho_{+}+\frac{B}{\rho_{+}^{\alpha}}\right)\right)=0, \\
& -\tau^{A}\left(\rho_{*}^{A} u_{*}^{A}-\rho_{+} u_{+}\right)+\left(\rho_{*}^{A} u_{*}^{A}\left(u_{*}^{A}-A \rho_{*}^{A}+\frac{B}{\left(\rho_{*}^{A}\right)^{\alpha}}\right)\right. \\
& \left.-\rho_{+} u_{+}\left(u_{+}-A \rho_{+}+\frac{B}{\rho_{+}^{\alpha}}\right)\right)=0 \text {, }
\end{aligned}
$$

from which and Lemma 7 we can easily get (68) and (70). 
8

Advances in Mathematical Physics

In what follows, we show the conclusion characterizing the limit of the solution to (1), (11) with initial data (12) for the case $\left(u_{+}, \rho_{+}\right) \in I I I\left(u_{-}, \rho_{-}\right)$as $A \rightarrow 0$.

Theorem 9. Let $\left(u_{+}, \rho_{+}\right) \in I I I\left(u_{-}, \rho_{-}\right)$and assume that $\left(u^{A}, \rho^{A}\right)(t, x)$ is the solution $S+J$ to Riemann problem (1), (11), and (12) for $0<A<A_{0}$ constructed in Section 3. Then

$$
\lim _{A \rightarrow 0} u^{A}(t, x)= \begin{cases}u_{-}, & x<\sigma_{0} t \\ u_{-}, & x=\sigma_{0} t \\ u_{+}, & x>\sigma_{0} t\end{cases}
$$

and $\rho^{A}$ and $\rho^{A} u^{A}$ converge in the sense of distributions, and the limit functions $\rho$ and $\rho u$ are the sums of a step function and $a$ Dirac delta function supported on $x=\sigma_{0} t$ with weights

$$
\begin{gathered}
\left(-\sigma_{0}[\rho]+\left[\rho\left(u+\frac{B}{\rho^{\alpha}}\right)\right]\right) t, \\
\left(-\sigma_{0}[\rho u]+\left[\rho u\left(u+\frac{B}{\rho^{\alpha}}\right)\right]\right) t,
\end{gathered}
$$

respectively.

Proof. (1) Set $\xi=x / t$. Then for each $A>0$, the Riemann solution $S+J$ can be expressed as

$$
\left(u^{A}, \rho^{A}\right)(\xi)= \begin{cases}\left(u_{-}, \rho_{-}\right), & \xi<\sigma^{A}, \\ \left(u_{*}^{A}, \rho_{*}^{A}\right), & \sigma^{A}<\xi<\tau^{A}, \\ \left(u_{+}, \rho_{+}\right), & \xi>\tau^{A}\end{cases}
$$

satisfying weak formulations

$$
\begin{aligned}
& -\int_{-\infty}^{+\infty} \rho^{A}\left(u^{A}-A \rho^{A}+\frac{B}{\left(\rho^{A}\right)^{\alpha}}-\xi\right) \phi^{\prime} d \xi \\
& +\int_{-\infty}^{+\infty} \rho^{A} \phi d \xi=0, \\
& -\int_{-\infty}^{+\infty} \rho^{A} u^{A}\left(u^{A}-A \rho^{A}+\frac{B}{\left(\rho^{A}\right)^{\alpha}}-\xi\right) \phi^{\prime} d \xi \\
& +\int_{-\infty}^{+\infty} \rho^{A} u^{A} \phi d \xi=0
\end{aligned}
$$

for any $\phi \in C_{0}^{1}(-\infty,+\infty)$. Equation (70) can be obtained easily.

(2) The first integral on the left-hand side of (73) can be decomposed into

$$
\begin{aligned}
& \int_{-\infty}^{+\infty} \rho^{A}\left(u^{A}-A \rho^{A}+\frac{B}{\left(\rho^{A}\right)^{\alpha}}-\xi\right) \phi^{\prime} d \xi \\
& =\left(\int_{-\infty}^{\sigma^{A}}+\int_{\sigma^{A}}^{\tau^{A}}+\int_{\tau^{A}}^{+\infty}\right) \rho^{A}\left(u^{A}-A \rho^{A}+\frac{B}{\left(\rho^{A}\right)^{\alpha}}-\xi\right) \phi^{\prime} d \xi .
\end{aligned}
$$

The sum of the first and last terms on the right-hand side of (75) is

$$
\begin{aligned}
& \int_{-\infty}^{\sigma^{A}} \rho^{A}\left(u^{A}-A \rho^{A}+\frac{B}{\left(\rho^{A}\right)^{\alpha}}-\xi\right) \phi^{\prime} d \xi \\
& \quad+\int_{\tau^{A}}^{+\infty} \rho^{A}\left(u^{A}-A \rho^{A}+\frac{B}{\left(\rho^{A}\right)^{\alpha}}-\xi\right) \phi^{\prime} d \xi \\
& =\int_{-\infty}^{\sigma^{A}} \rho_{-}\left(u_{-}-A \rho_{-}+\frac{B}{\rho_{-}^{\alpha}}-\xi\right) \phi^{\prime} d \xi \\
& \quad+\int_{\tau^{A}}^{+\infty} \rho_{+}\left(u_{+}-A \rho_{+}+\frac{B}{\rho_{+}^{\alpha}}-\xi\right) \phi^{\prime} d \xi \\
& =-\rho_{-} \sigma^{A} \phi\left(\sigma^{A}\right)+\rho_{+} \tau^{A} \phi\left(\tau^{A}\right) \\
& \quad+\rho_{-}\left(u_{-}-A \rho_{-}+\frac{B}{\rho_{-}^{\alpha}}\right) \phi\left(\tau^{A}\right) \\
& \quad-\rho_{+}\left(u_{+}-A \rho_{+}+\frac{B}{\rho_{+}^{\alpha}}\right) \phi\left(\sigma^{A}\right) \\
& +\int_{-\infty}^{\sigma^{A}} \rho_{-} \phi d \xi+\int_{\tau^{A}}^{+\infty} \rho_{+} \phi d \xi,
\end{aligned}
$$

which converges as $A \rightarrow 0$ to

$$
\begin{gathered}
\left(-\sigma_{0}[\rho]+\left[\rho\left(u+\frac{B}{\rho^{\alpha}}\right)\right]\right) \phi\left(\sigma_{0}\right) \\
+\int_{-\infty}^{+\infty} H\left(\xi-\sigma_{0}\right) \phi(\xi) d \xi
\end{gathered}
$$

with

$$
H(x)= \begin{cases}\rho_{-}, & x<0, \\ \rho_{+}, & x>0 .\end{cases}
$$

The second term on the right hand of (75) is

$$
\begin{gathered}
\int_{\sigma^{A}}^{\tau^{A}} \rho^{A}\left(u^{A}-A \rho^{A}+\frac{B}{\left(\rho^{A}\right)^{\alpha}}-\xi\right) \phi^{\prime} d \xi \\
=\int_{\sigma^{A}}^{\tau^{A}} \rho_{*}^{A}\left(u_{*}^{A}-A \rho_{*}^{A}+\frac{B}{\left(\rho_{*}^{A}\right)^{\alpha}}-\xi\right) \phi^{\prime} d \xi \\
=\rho_{*}^{A}\left(\tau^{A}-\sigma^{A}\right)\left(\frac{\phi\left(\tau^{A}\right)-\phi\left(\sigma^{A}\right)}{\tau^{A}-\sigma^{A}}\left(u_{*}^{A}-A \rho_{*}^{A}+\frac{B}{\left(\rho_{*}^{A}\right)^{\alpha}}\right)\right. \\
-\frac{\tau^{A} \phi\left(\tau^{A}\right)-\sigma_{-}^{A} \phi\left(\sigma^{A}\right)}{\tau^{A}-\sigma^{A}} \\
\left.+\frac{1}{\tau^{A}-\sigma^{A}} \int_{\sigma^{A}}^{\tau^{A}} \phi d \xi\right),
\end{gathered}
$$

which converges as $A \rightarrow 0$ to

$$
\begin{aligned}
& \left(-\sigma_{0}[\rho]+\left[\rho\left(u+\frac{B}{\rho^{\alpha}}\right)\right]\right) \\
& \quad \times\left(\sigma_{0} \phi^{\prime}\left(\sigma_{0}\right)-\sigma_{0} \phi^{\prime}\left(\sigma_{0}\right)-\phi\left(\sigma_{0}\right)+\phi\left(\sigma_{0}\right)\right)=0 .
\end{aligned}
$$




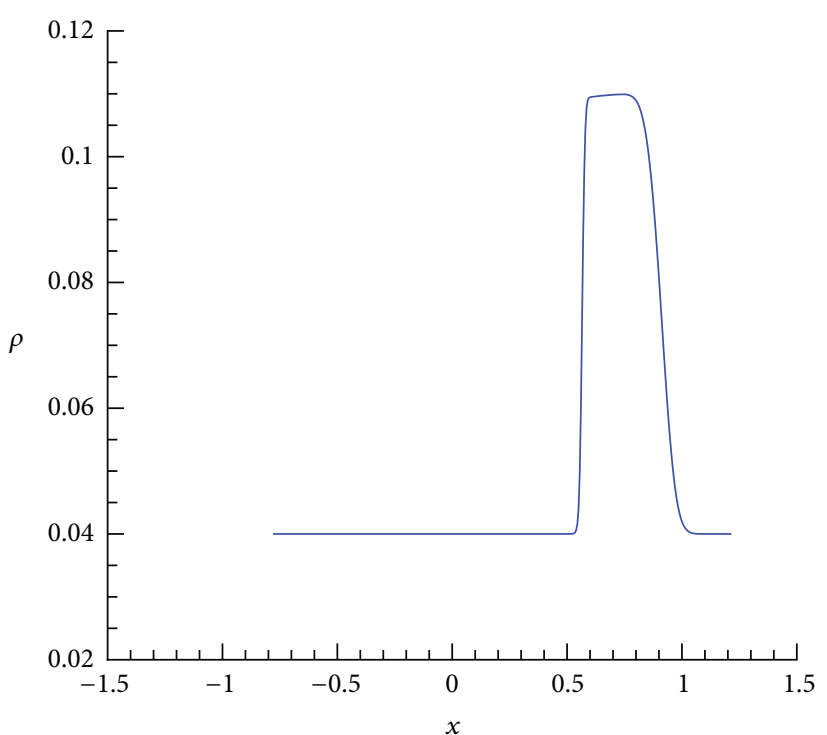

(a)

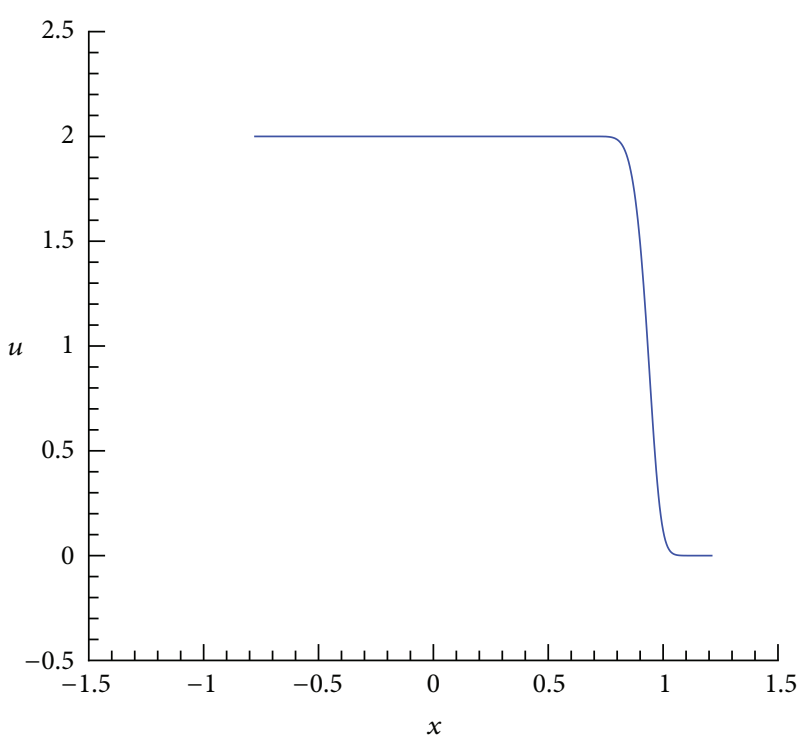

(b)

FIgURE 4: Numerical results for $S+J$.

Returning to (73), we immediately obtain that

$$
\begin{aligned}
\lim _{A \rightarrow 0} & \int_{-\infty}^{+\infty}\left(\rho^{A}-H\left(\xi-\sigma_{0}\right)\right) \phi d \xi \\
& =\left(-\sigma_{0}[\rho]+\left[\rho\left(u+\frac{B}{\rho^{\alpha}}\right)\right]\right) \phi\left(\sigma_{0}\right) .
\end{aligned}
$$

Similarly, from (74), we can obtain

$$
\begin{aligned}
\lim _{A \rightarrow 0} & \int_{-\infty}^{+\infty}\left(\rho^{A} u^{A}-\widetilde{H}\left(\xi-\sigma_{0}\right)\right) \phi d \xi \\
& =\left(-\sigma_{0}[\rho u]+\left[\rho u\left(u+\frac{B}{\rho^{\alpha}}\right)\right]\right) \phi\left(\sigma_{0}\right)
\end{aligned}
$$

with

$$
\widetilde{H}(x)= \begin{cases}\rho_{-} u_{-}, & x<0, \\ \rho_{+} u_{+}, & x>0 .\end{cases}
$$

(3) We study the limits of $\rho^{A}$ and $\rho^{A} u^{A}$ by tracking the time-dependence of the weights of the $\delta$-measure as $A \rightarrow 0$. Taking (81) into account, we have for any $\psi \in C_{0}^{\infty}\left(R \times R^{+}\right)$

$$
\begin{aligned}
\lim _{A \rightarrow 0} \int_{0}^{+\infty} \int_{-\infty}^{+\infty} \rho^{A}\left(\frac{x}{t}\right) \psi(x, t) d x d t \\
=\lim _{A \rightarrow 0} \int_{0}^{+\infty}\left(\int_{-\infty}^{+\infty} \rho^{A}\left(\frac{x}{t}\right) \psi(x, t) d x\right) d t \\
=\lim _{A \rightarrow 0} \int_{0}^{+\infty}\left(\int_{-\infty}^{+\infty} \rho^{A}(\xi) \psi(\xi t, t) d(\xi t)\right) d t \\
=\lim _{A \rightarrow 0} \int_{0}^{+\infty} t\left(\int_{-\infty}^{+\infty} \rho^{A}(\xi) \psi(\xi t, t) d \xi\right) d t \\
=\int_{0}^{+\infty} t\left(\left(-\sigma_{0}[\rho]+\left[\rho\left(u+\frac{B}{\rho^{\alpha}}\right)\right]\right) \psi\left(\sigma_{0} t, t\right)\right. \\
\left.\quad+\int_{-\infty}^{+\infty} H\left(\xi-\sigma_{0}\right) \psi(\xi t, t) d \xi\right) d t
\end{aligned}
$$

$$
\begin{aligned}
= & \int_{0}^{+\infty}\left(-\sigma_{0}[\rho]+\left[\rho\left(u+\frac{B}{\rho^{\alpha}}\right)\right]\right) t \psi\left(\sigma_{0} t, t\right) d t \\
& +\int_{0}^{+\infty} t\left(\int_{-\infty}^{+\infty} H\left(\xi-\sigma_{0}\right) \psi(\xi t, t) d \xi\right) d t \\
= & \int_{0}^{+\infty}\left(-\sigma_{0}[\rho]+\left[\rho\left(u+\frac{B}{\rho^{\alpha}}\right)\right]\right) t \psi\left(\sigma_{0} t, t\right) d t \\
& +\int_{0}^{+\infty} \int_{-\infty}^{+\infty} H\left(x-\sigma_{0} t\right) \psi(x, t) d x d t,
\end{aligned}
$$

in which by (31)

$$
\begin{aligned}
\int_{0}^{+\infty} & \left(-\sigma_{0}[\rho]+\left[\rho\left(u+\frac{B}{\rho^{\alpha}}\right)\right]\right) t \psi\left(\sigma_{0} t, t\right) d t \\
& =\left\langle w_{1}(\cdot) \delta_{L}, \psi(\cdot, \cdot)\right\rangle
\end{aligned}
$$

with

$$
w_{1}(t)=\left(-\sigma_{0}[\rho]+\left[\rho\left(u+\frac{B}{\rho^{\alpha}}\right)\right]\right) t .
$$

Similarly, from (82), we have for any $\psi \in C_{0}^{\infty}\left(R \times R^{+}\right)$

$$
\begin{aligned}
& \lim _{A \rightarrow 0} \int_{0}^{+\infty} \int_{-\infty}^{+\infty} \rho^{A}\left(\frac{x}{t}\right) \psi(x, t) d x d t \\
& =\int_{0}^{+\infty}\left(-\sigma_{0}[\rho u]+\left[\rho u\left(u+\frac{B}{\rho^{\alpha}}\right)\right]\right) t \psi\left(\sigma_{0} t, t\right) d t \\
& \quad+\int_{0}^{+\infty} \int_{-\infty}^{+\infty} \widetilde{H}\left(x-\sigma_{0} t\right) \psi(x, t) d x d t
\end{aligned}
$$

in which

$$
\begin{aligned}
\int_{0}^{+\infty} & \left(-\sigma_{0}[\rho u]+\left[\rho u\left(u+\frac{B}{\rho^{\alpha}}\right)\right]\right) t \psi\left(\sigma_{0} t, t\right) d t \\
& =\left\langle w_{2}(\cdot) \delta_{L}, \psi(\cdot, \cdot)\right\rangle
\end{aligned}
$$




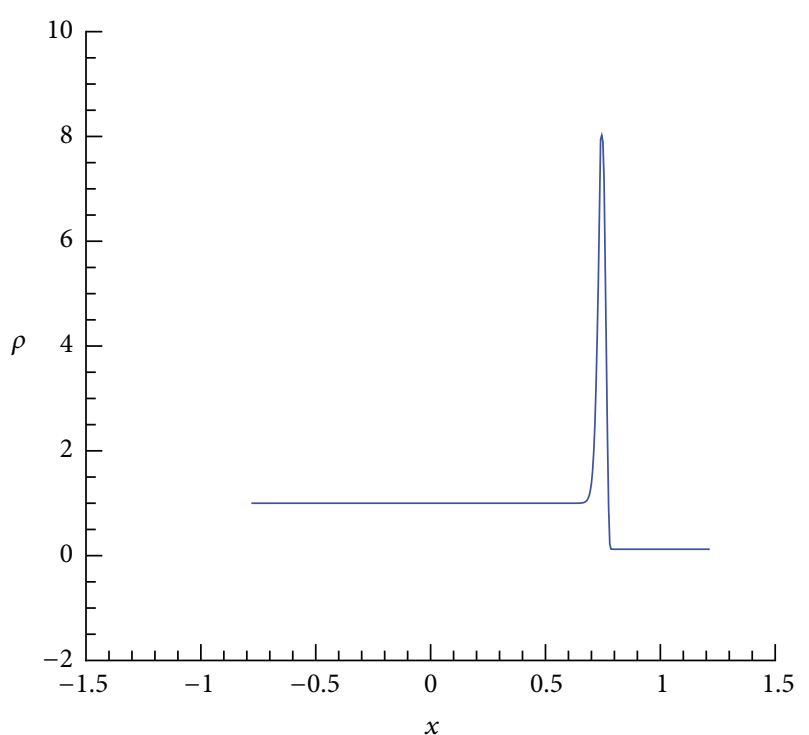

(a)

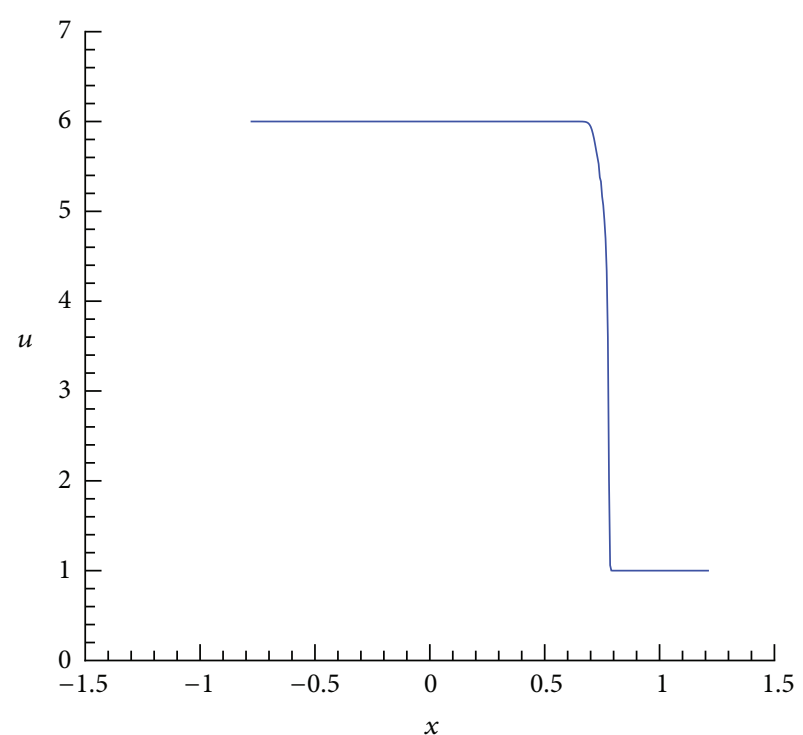

(b)

FiguRE 5: Numerical results for $\delta$.

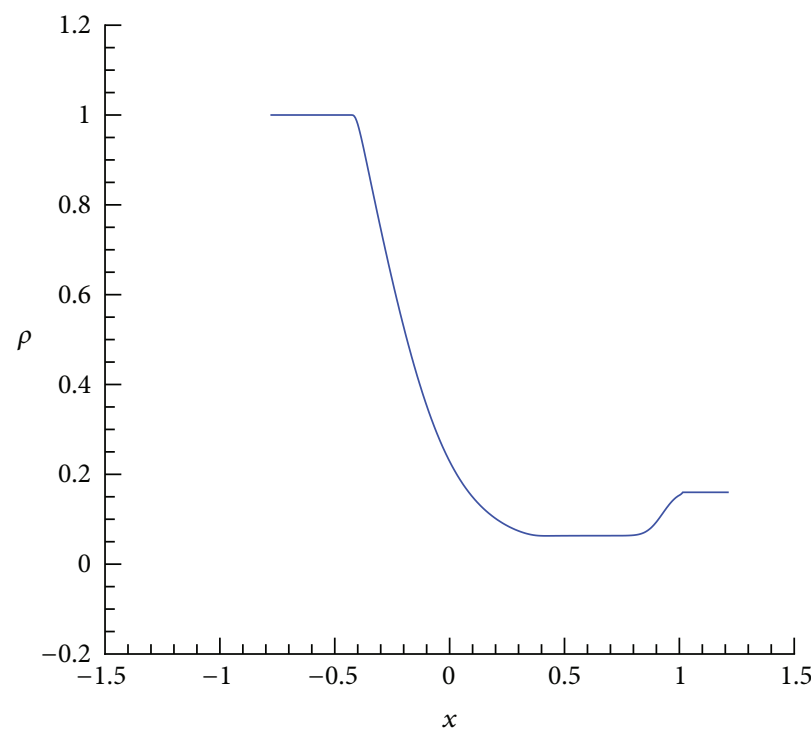

(a)

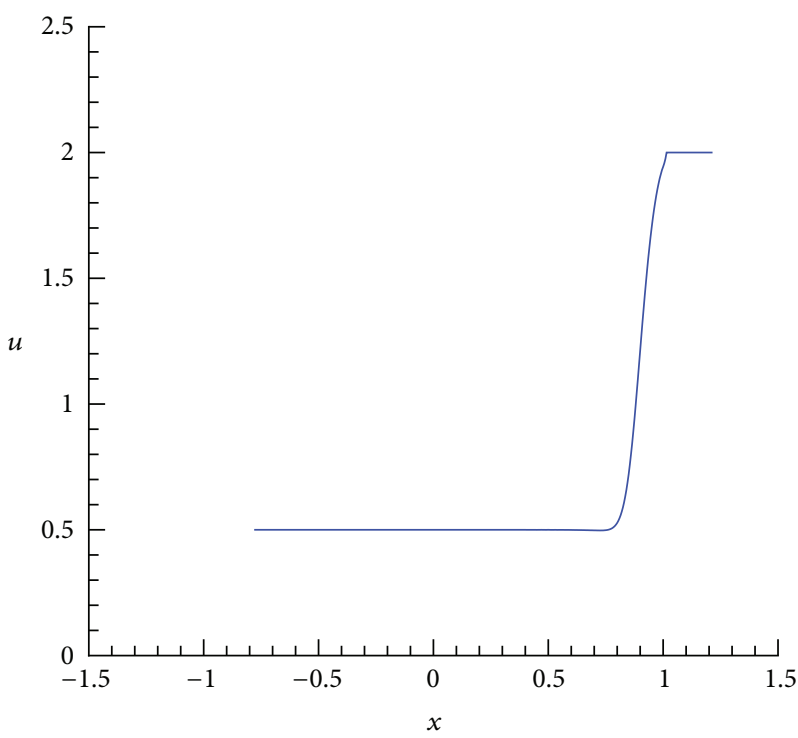

(b)

FIgURE 6: Numerical results for $R+J$.

with

$$
w_{2}(t)=\left(-\sigma_{0}[\rho u]+\left[\rho u\left(u+\frac{B}{\rho^{\alpha}}\right)\right]\right) t
$$

This completes the proof.

One can observe that when $u_{+}+\left(B / \rho_{+}^{\alpha}\right)<u_{-}$, the weight and speed of the delta shock wave in the limit situation are different from the weight and speed of the delta shock wave obtained by solving (1), (10) with the same initial data (11) in Section 2; and only when $u_{+}+\left(B / \rho_{+}^{\alpha}\right)=u_{-}$, they are coincident.

\section{Numerical Simulations}

In this section, we simulate the Riemann solutions obtained in Sections 2 and 3 and examine the processes of formation of delta shock waves studied in Section 4. To discretize the system, we employ the Nessyahu-Tadmor scheme [20] with $500 \times 500$ cells and CFL $=0.475 . \alpha=0.5$ and $B=1$ are taken. 


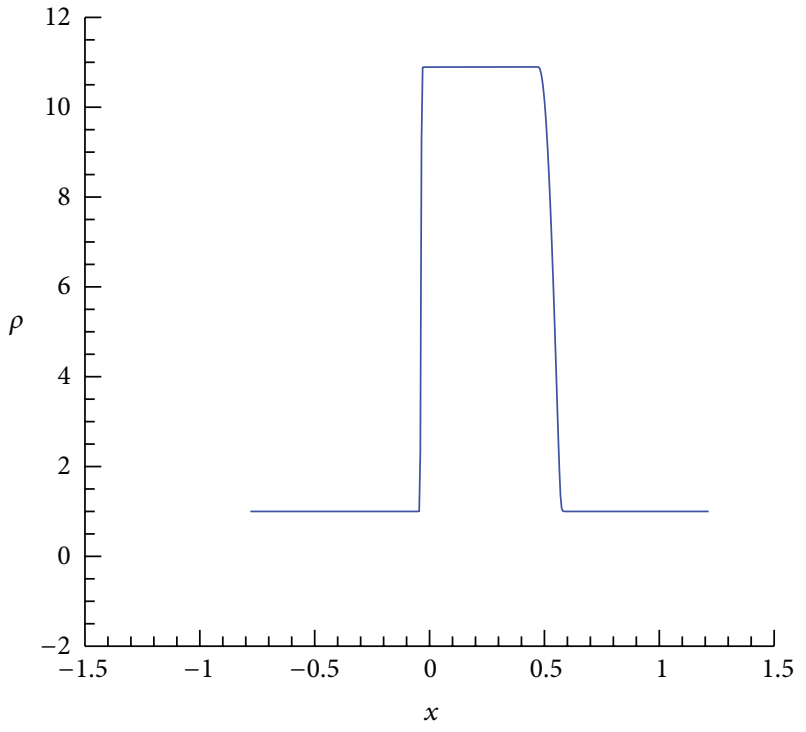

(a)

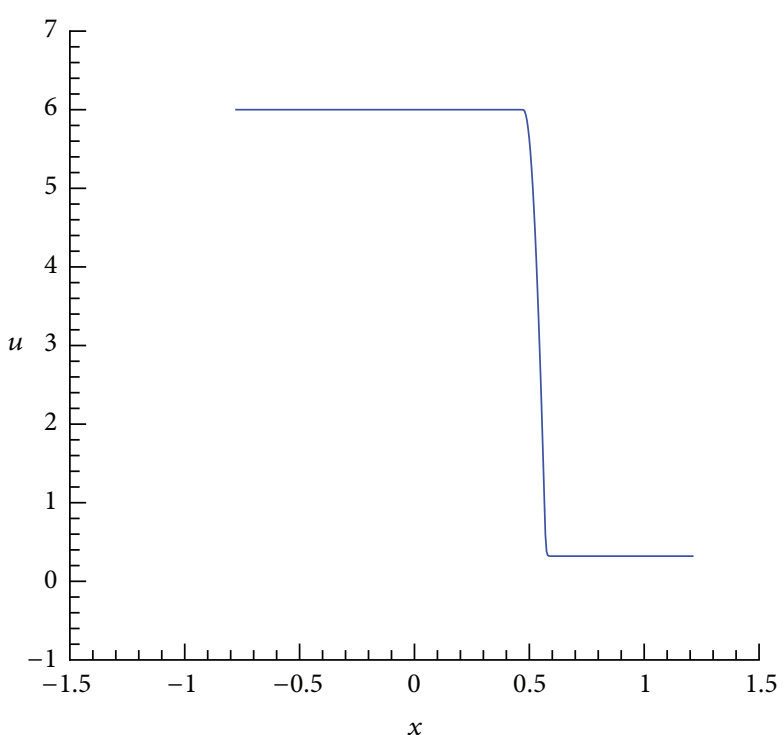

(b)

Figure 7: Numerical results for $S+J$.

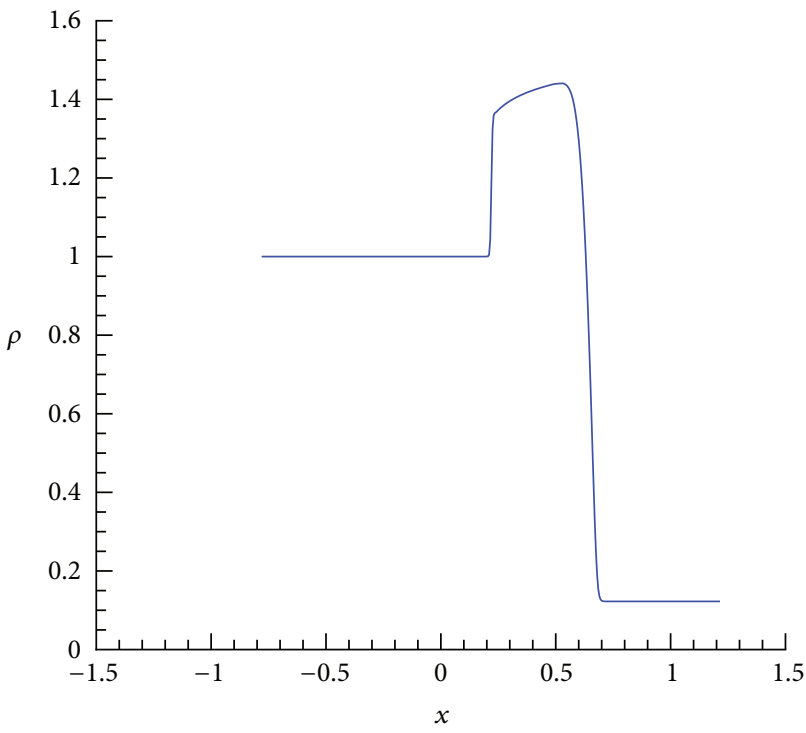

(a)

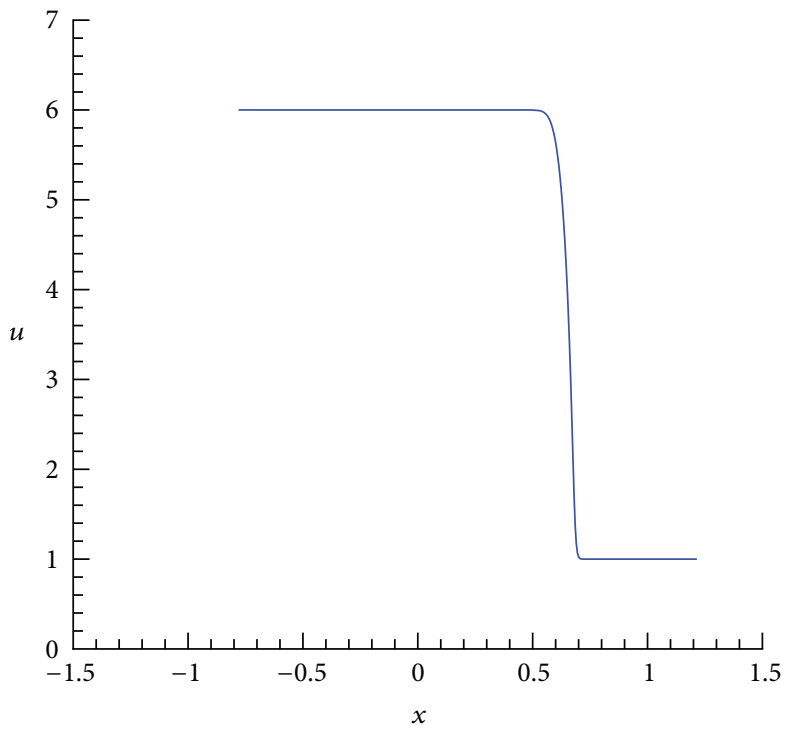

(b)

FIGURE 8: Numerical results for $A=2$.

5.1. Numerical Simulations of Solutions to (1) and (11) with (10)

Case $1(R+J)$. For this case, we take the initial data $\left(u_{-}, \rho_{-}\right)=$ $(0.5,1.0)$ and $\left(u_{+}, \rho_{+}\right)=(2.0,0.16)$. The numerical results at $t=0.3$ are shown in Figure 3 .

Case $2(S+J)$. In this case, the initial data are taken as $\left(u_{-}, \rho_{-}\right)=(2.0,0.04)$ and $\left(u_{+}, \rho_{+}\right)=(0.0,0.04)$. The numerical results at $t=0.3$ are presented in Figure 4 .

Case $3(\delta)$. At this moment, the initial data are taken as $\left(u_{-}, \rho_{-}\right)=(6.0,1.0)$ and $\left(u_{+}, \rho_{+}\right)=(1.0,0.1225)$. The numerical results at $t=0.2$ are given in Figure 5 .
One can observe that all the numerical results are in complete agreement with the theoretical analysis.

5.2. Numerical Simulations of Solutions to (1) and (11) with (12)

Case $1(R+J)$. For this case, we take the initial data $\left(u_{-}, \rho_{-}\right)=$ $(0.5,1.0)$ and $\left(u_{+}, \rho_{+}\right)=(2.0,0.16)$. The numerical results with $A=0.5$ at $t=0.3$ are shown in Figure 6 .

Case $2(S+J)$. In this case, the initial data are taken as $\left(u_{-}, \rho_{-}\right)=(6.0,1.0)$ and $\left(u_{+}, \rho_{+}\right)=(0.32,1.0)$. The numerical results with $A=0.5$ at $t=1$ are presented in Figure 7 . 


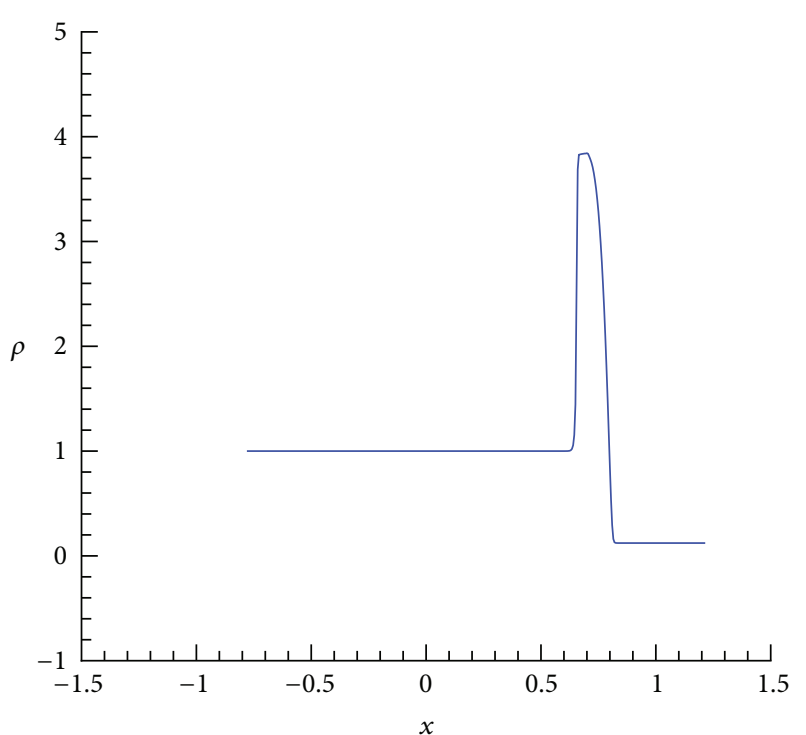

(a)

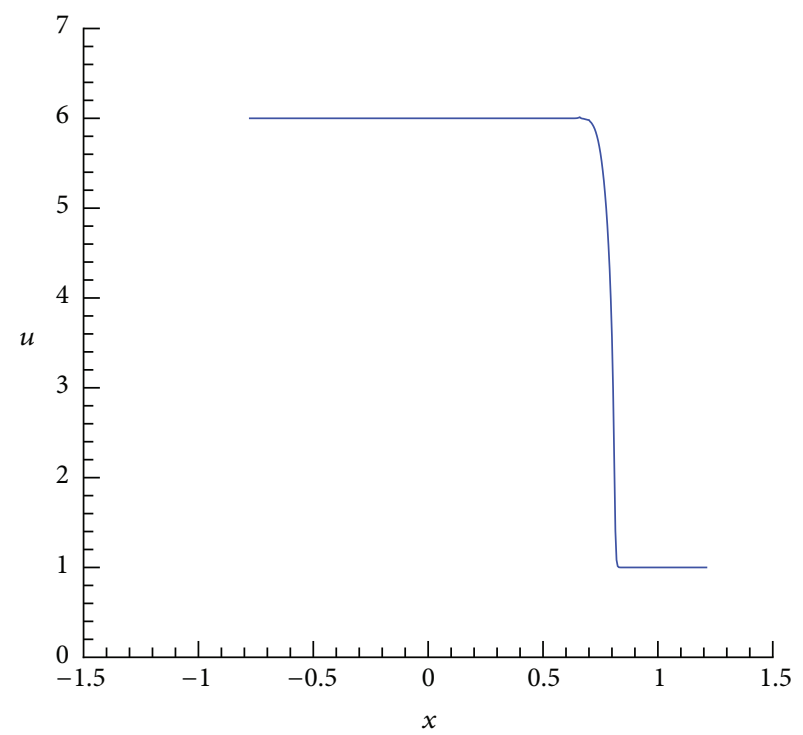

(b)

Figure 9: Numerical results for $A=0.5$.

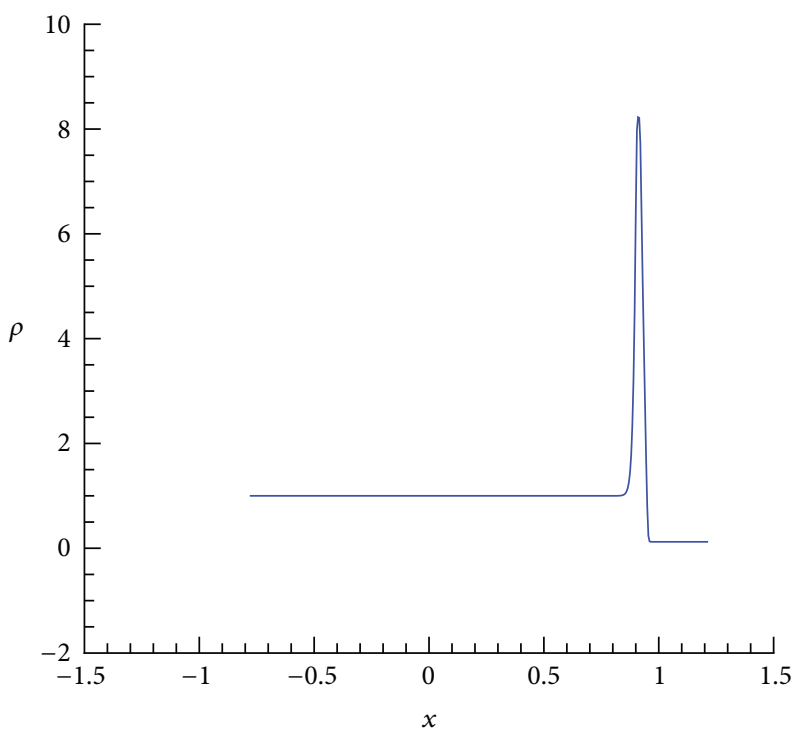

(a)

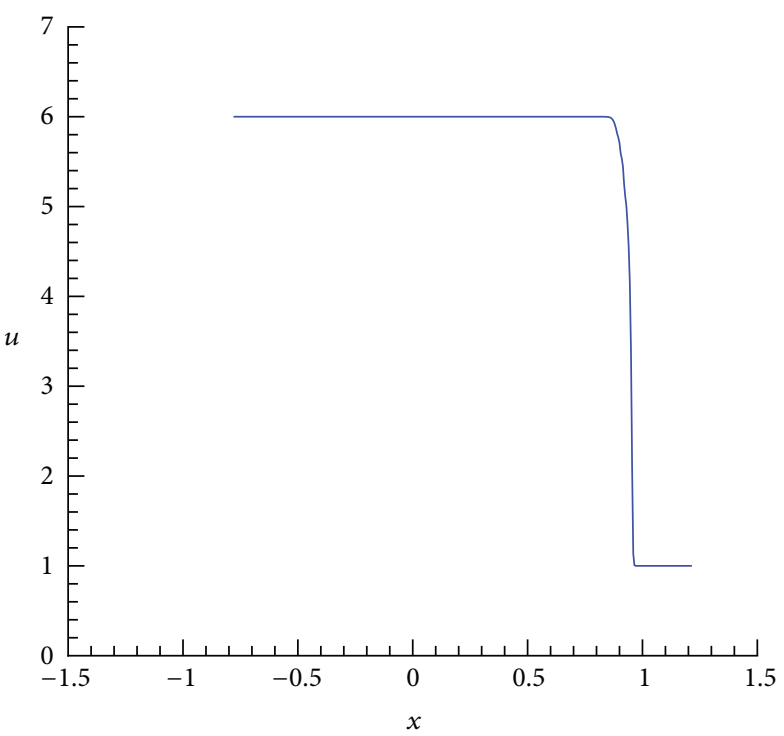

(b)

Figure 10: Numerical results for $A=0.05$.

It can be seen that these numerical results are also in accord with the theoretical analysis.

5.3. Numerical Simulations of Formation Process of Delta Shock Waves. We take the initial data $\left(u_{-}, \rho_{-}\right)=(6.0,1.0)$ and $\left(u_{+}, \rho_{+}\right)=(1.0,0.1225)$. The numerical results corresponding to different $A$ at $t=0.2$ are given in Figures 8,9 , and 10 .

One can see clearly from these numerical results that when $A$ decreases, the location of the shock and contact discontinuity becomes closer, and the density of the intermediate state increases dramatically.

\section{Conclusion}

The nonlinear hyperbolic systems of conservation laws of Keyfitz-Kranzer type in the symmetric or nonsymmetric form are important models. This paper is concerned with a special nonsymmetric Keyfitz-Kranzer system (1) with an unknown function $p(\rho)$, which is often encountered in some problems. Generally, its solution depends strongly on the behaviors of $p(\rho)$. It is very necessary to study such a system for different choices of $p(\rho)$.

Previously, the investigations are focused on the polytropic gas pressure law $p=\rho^{\gamma}, \gamma>0[15,16]$. Recently, 
TABLE 1: Waves involved in the Riemann solutions for different $p(\rho)$.

\begin{tabular}{llllll}
\hline & $R$ & $S$ & $J$ & Vac & $\delta$ \\
\hline PG & $\checkmark$ & $\checkmark$ & $\checkmark$ & $\checkmark$ & $\boldsymbol{x}$ \\
CG & $\boldsymbol{x}$ & $\boldsymbol{x}$ & $\checkmark$ & $\boldsymbol{x}$ & $\checkmark$ \\
GCG & $\checkmark$ & $\checkmark$ & $\checkmark$ & $\boldsymbol{x}$ & $\checkmark$ \\
MCG & $\checkmark$ & $\checkmark$ & $\checkmark$ & $\boldsymbol{x}$ & $\boldsymbol{x}$ \\
\hline
\end{tabular}

Cheng [19] introduced the Chaplygin gas pressure law (8). In the present paper, we consider the generalized Chaplygin gas pressure law (10) and the modified Chaplygin gas pressure law (12).

It is well known that, for nonlinear conservation laws, the classical waves consist of rarefaction wave $(R)$, shock $(S)$, contact discontinuity $(J)$, and vacuum (Vac), and the nonclassical ones contain delta shock wave $(\delta)$. For system (1), we find that the waves involved in the Riemann solutions are different for different choices of the function $p(\rho)$. This fact is shown in Table 1, where PG, CG, GCG, and MCG denote the pressure law for polytropic gas, standard Chaplygin gas, generalized Chaplygin gas, and modified Chaplygin gas, respectively; $\checkmark$ means that the waves will appear in the Riemann solutions, and $\boldsymbol{X}$ is opposite. It is also concluded that only when the domain $I I I\left(u_{-}, \rho_{-}\right)$appears in the phase plane, delta shock wave will develop.

It is seen that the modified Chaplygin gas pressure law can be regarded as a linear combination of the polytropic gas pressure law (the case for $\gamma=1$ ) and the generalized Chaplygin gas pressure law. We are interested in the limits of the Riemann solutions for the modified Chaplygin gas pressure law as parameter $A$ vanishes. As a result, it is in particular proved that, for some initial data, the limit is a delta shock wave. However, it is not coincident with the delta shock solution to the limit system. This is different from the results in $[7,8]$.

\section{Acknowledgments}

The authors would like to thank the anonymous referees for providing valuable suggestions and pointing out imprecise expressions. The research Supported by the National Natural Science Foundation of China $(11226191,10961025)$.

\section{References}

[1] H. C. Kranzer and B. L. Keyfitz, "A strictly hyperbolic system of conservation laws admitting singular shocks," in Nonlinear Evolution Equations that Change Type, vol. 27, pp. 107-125, Springer, New York, NY, USA, 1990.

[2] P. LeFloch, "An existence and uniqueness result for two nonstrictly hyperbolic systems," in Nonlinear Evolution Equations that Change Type, vol. 27, pp. 126-138, Springer, New York, NY, USA, 1990.

[3] D. C. Tan and T. Zhang, “Two-dimensional Riemann problem for a hyperbolic system of nonlinear conservation laws I. Four-J cases, II," Journal of Differential Equations, vol. 111, no. 2, pp. 203-254, 1994.
[4] D. C. Tan, T. Zhang, and Y. X. Zheng, "Delta-shock waves as limits of vanishing viscosity for hyperbolic systems of conservation laws," Journal of Differential Equations, vol. 112, no. 1, pp. 1-32, 1994.

[5] J. Li, T. Zhang, and S. Yang, The Two-Dimensional Riemann Problem in Gas Dynamics, vol. 98 of Pitman Monographs and Surveys in Pure and Applied Mathematics, Longman, 1998.

[6] H. Yang, "Riemann problems for a class of coupled hyperbolic systems of conservation laws," Journal of Differential Equations, vol. 159, no. 2, pp. 447-484, 1999.

[7] G.-Q. Chen and H. Liu, "Formation of $\delta$-shocks and vacuum states in the vanishing pressure limit of solutions to the Euler equations for isentropic fluids," SIAM Journal on Mathematical Analysis, vol. 34, no. 4, pp. 925-938, 2003.

[8] G.-Q. Chen and H. Liu, "Concentration and cavitation in the vanishing pressure limit of solutions to the Euler equations for nonisentropic fluids," Physica D, vol. 189, no. 1-2, pp. 141-165, 2004.

[9] V. M. Shelkovich, "The Riemann problem admitting $\delta$-, $\delta^{\prime}$-shocks, and vacuum states (the vanishing viscosity approach)," Journal of Differential Equations, vol. 231, no. 2, pp. 459-500, 2006.

[10] L. Guo, W. Sheng, and T. Zhang, "The two-dimensional Riemann problem for isentropic Chaplygin gas dynamic system," Communications on Pure and Applied Analysis, vol. 9, no. 2, pp. 431-458, 2010.

[11] H. Cheng and H. Yang, "Delta shock waves in chromatography equations," Journal of Mathematical Analysis and Applications, vol. 380, no. 2, pp. 475-485, 2011.

[12] H. Cheng and H. Yang, "Riemann problem for the isentropic relativistic Chaplygin Euler equations," Zeitschrift für Angewandte Mathematik und Physik, vol. 63, no. 3, pp. 429-440, 2012.

[13] H. Yang and Y. Zhang, "New developments of delta shock waves and its applications in systems of conservation laws," Journal of Differential Equations, vol. 252, no. 11, pp. 5951-5993, 2012.

[14] B. L. Keyfitz and H. C. Kranzer, "A system of nonstrictly hyperbolic conservation laws arising in elasticity theory," Archive for Rational Mechanics and Analysis, vol. 72, no. 3, pp. 219-241, 1980.

[15] Y.-g. Lu, "Existence of global bounded weak solutions to nonsymmetric systems of Keyfitz-Kranzer type," Journal of Functional Analysis, vol. 261, no. 10, pp. 2797-2815, 2011.

[16] A. Aw and M. Rascle, "Resurrection of "second order" models of traffic flow," SIAM Journal on Applied Mathematics, vol. 60, no. 3, pp. 916-938, 2000.

[17] P. D. Lax, "Hyperbolic systems of conservation laws. II," Communications on Pure and Applied Mathematics, vol. 10, pp. 537566, 1957.

[18] P. D. Lax, Hyperbolic Systems of Conservation Laws and the Mathematical Theory of Shock Waves, vol. 11 of CBMS Regional Conference Series in Applied Mathematics, Society for industrial and Applied Mathematics, Philadelphia, 1973.

[19] H. Cheng, "Delta shock waves for a linearly degenerate hyperbolic system of conservation laws of Keyfitz-Kranzer type," Advances in Mathematical Physics, vol. 2013, Article ID 958120, 10 pages, 2013.

[20] H. Nessyahu and E. Tadmor, "Nonoscillatory central differencing for hyperbolic conservation laws," Journal of Computational Physics, vol. 87, no. 2, pp. 408-463, 1990.

[21] S. A. Chaplygin, "On gas jets," Scientific Memoirs, Moscow University Mathematic Physics, vol. 21, no. 1063, pp. 1-121, 1904. 
[22] H.-S. Tsien, "Two-dimensional subsonic flow of compressible fluids," Journal of the Aeronautical Sciences, vol. 6, pp. 399-407, 1939.

[23] M. Bento, O. Bertolami, and A. Sen, "Generalized Chaplygin gas, accelerated expansion and dark energy-matter unification," Physical Review D, vol. 66, Article ID 043507, 5 pages, 2002.

[24] H. Benaoum, "Accelerated universe from modified Chaplygin gas and tachyonic fluid," High Energy Physics, 11 pages, 2002, http://arxiv.org/abs/hep-th/0205140.

[25] H. B. Benaoum, "Modified Chaplygin gas cosmology," Advances in High Energy Physics, Article ID 357802, 12 pages, 2012.

[26] Y. Brenier, "Solutions with concentration to the Riemann problem for the one-dimensional Chaplygin gas equations," Journal of Mathematical Fluid Mechanics, vol. 7, no. suppl. 3, pp. S326S331, 2005. 


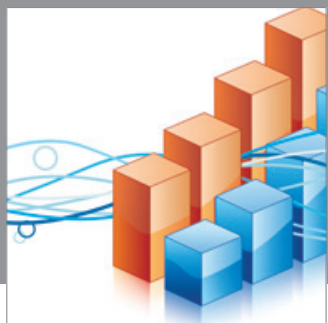

Advances in

Operations Research

mansans

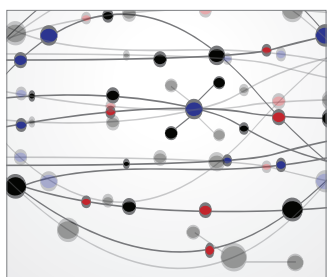

The Scientific World Journal
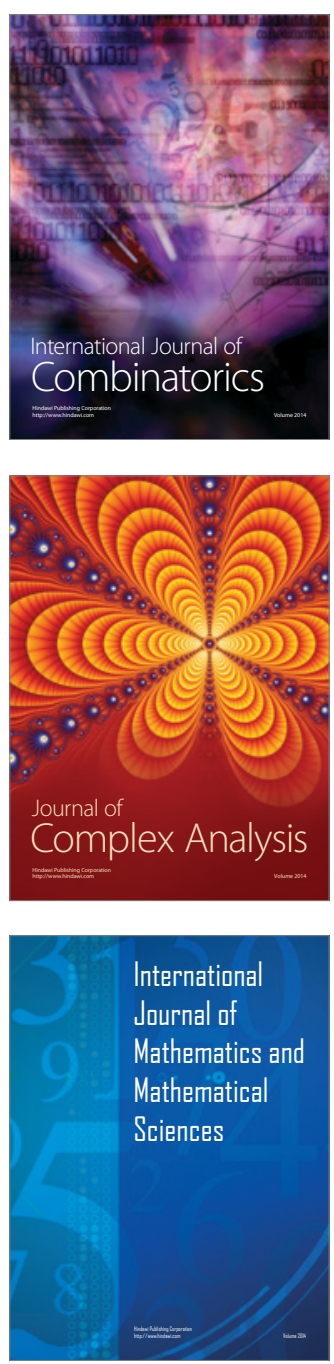
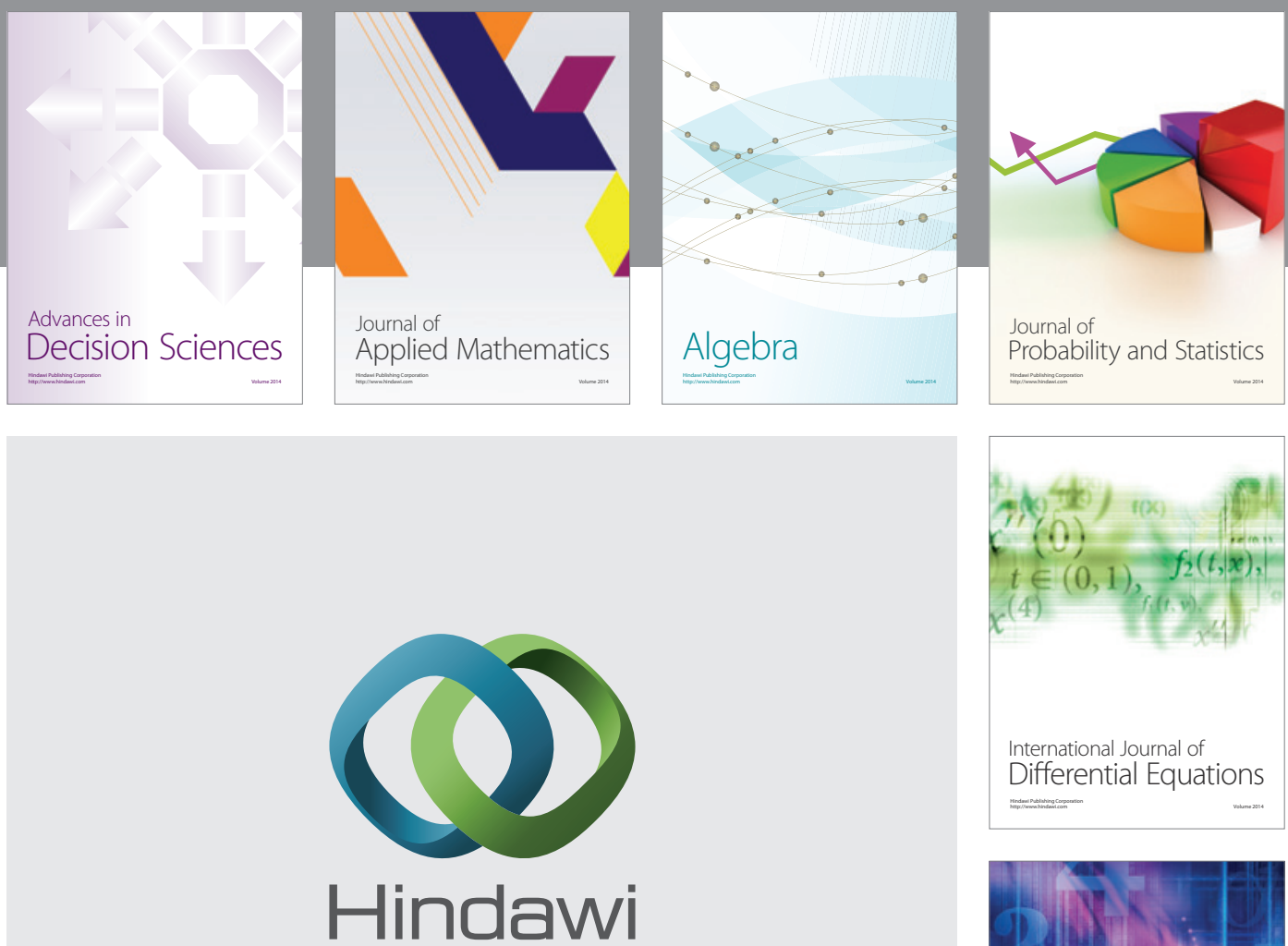

Submit your manuscripts at http://www.hindawi.com
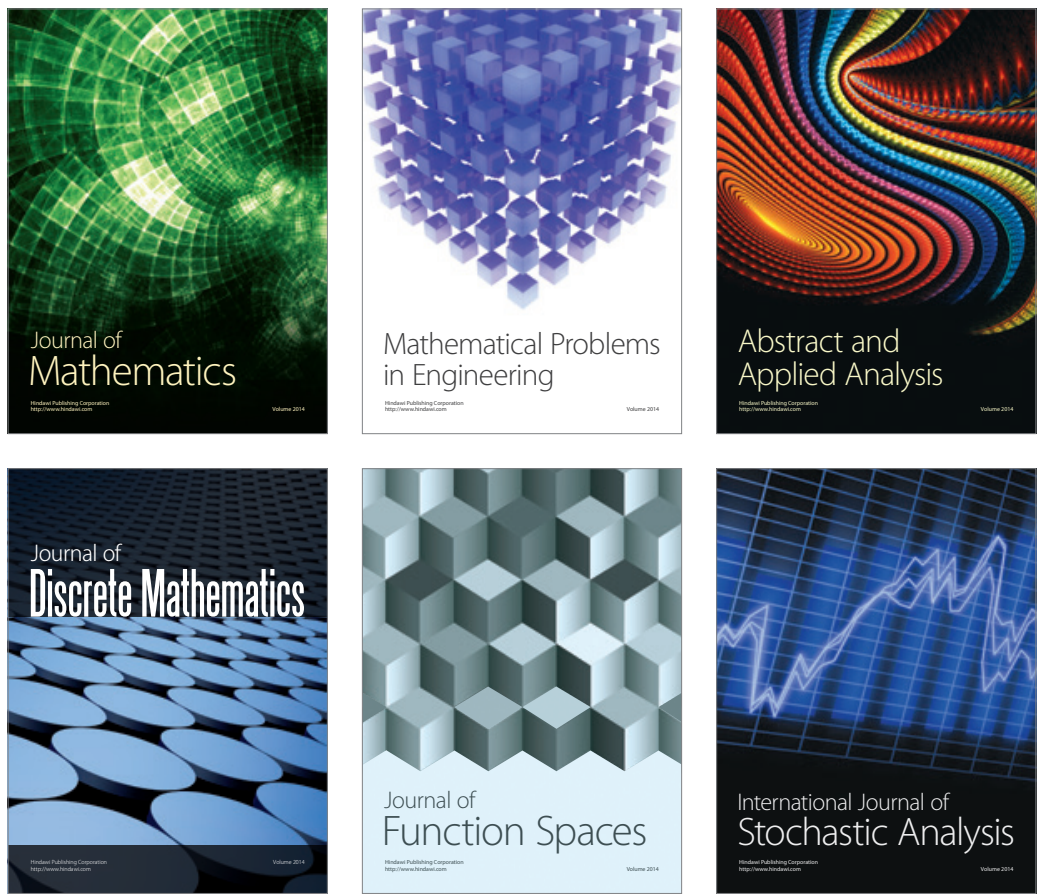

Journal of

Function Spaces

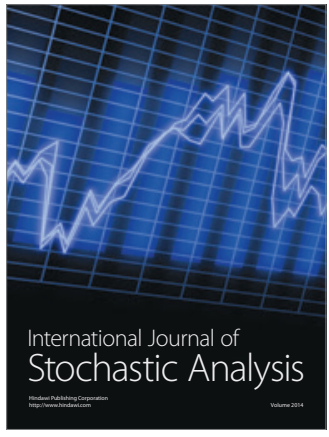

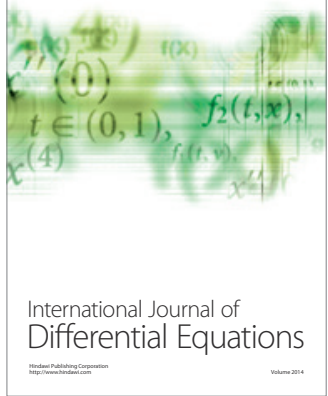
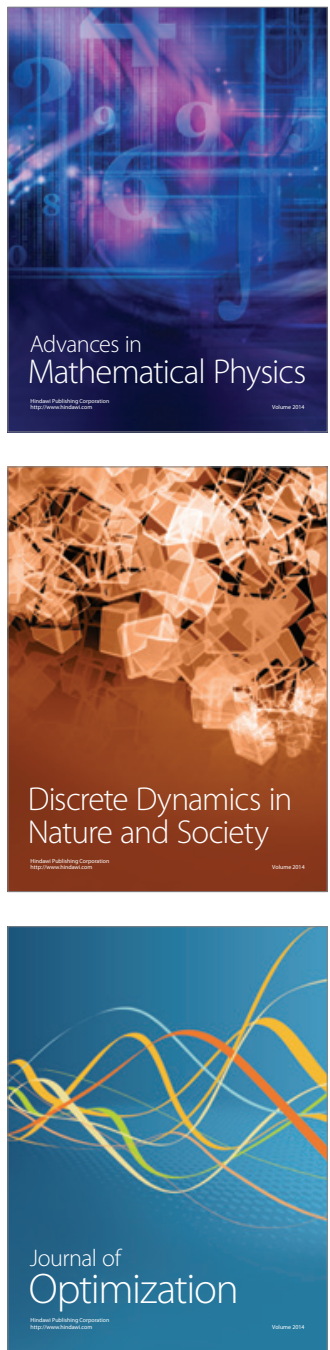